\title{
Effect of dislocations on electrical and electron transport properties of InN thin films. I. Strain relief and formation of a dislocation network
}

Cite as: J. Appl. Phys. 100, 094902 (2006); https://doi.org/10.1063/1.2363233

Submitted: 19 February 2006 . Accepted: 21 September 2006 . Published Online: 07 November 2006

V. Lebedev, V. Cimalla, J. Pezoldt, M. Himmerlich, S. Krischok, J. A. Schaefer, O. Ambacher, F. M. Morales, J. G. Lozano, D. González, et al.

\section{ARTICLES YOU MAY BE INTERESTED IN}

Effect of dislocations on electrical and electron transport properties of InN thin films. II. Density and mobility of the carriers

Journal of Applied Physics 100, 094903 (2006); https://doi.org/10.1063/1.2363234

Indium nitride (InN): A review on growth, characterization, and properties

Journal of Applied Physics 94, 2779 (2003); https://doi.org/10.1063/1.1595135

Threading dislocations in In-polar InN films and their effects on surface morphology and electrical properties

Applied Physics Letters 90, 151901 (2007); https://doi.org/10.1063/1.2720717

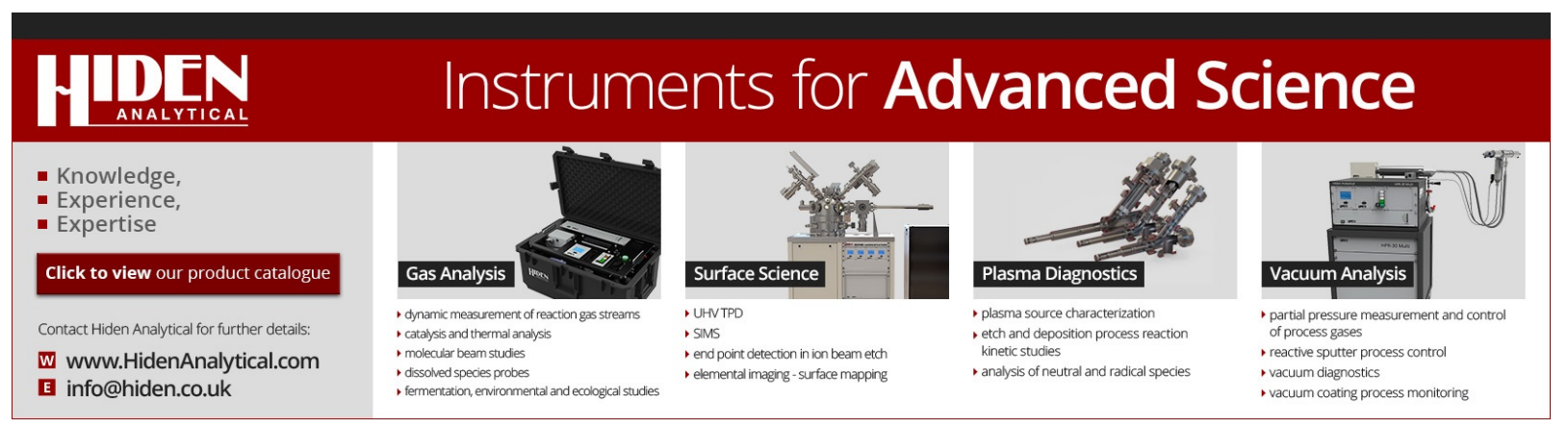




\title{
Effect of dislocations on electrical and electron transport properties of InN thin films. I. Strain relief and formation of a dislocation network
}

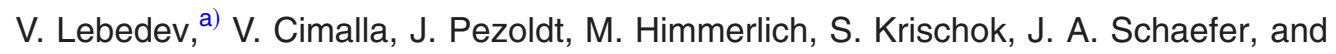 \\ O. Ambacher \\ Center for Micro- and Nanotechnologies, Technical University Ilmenau, D-98684 Ilmenau, Germany \\ F. M. Morales, ${ }^{\text {b) }}$ J. G. Lozano, and D. González \\ Departamento de Ciencia de los Materiales e Ingeniería Metalúrgica y Química Inorgánica, \\ Facultad de Ciencias, Universidad de Cádiz, 11510 Puerto Real Cádiz, España
}

(Received 19 February 2006; accepted 21 September 2006; published online 7 November 2006)

\begin{abstract}
The strain-relaxation phenomena and the formation of a dislocation network in $2 \mathrm{H}$ - $\mathrm{InN}$ epilayers during molecular beam epitaxy are reported. Plastic and elastic strain relaxations were studied by reflection high-energy electron diffraction, transmission electron microscopy, and high resolution x-ray diffraction. Characterization of the surface properties has been performed using atomic force microscopy and photoelectron spectroscopy. In the framework of the growth model the following stages of the strain relief have been proposed: plastic relaxation of strain by the introduction of geometric misfit dislocations, elastic strain relief during island growth, formation of threading dislocations induced by the coalescence of the islands, and relaxation of elastic strain by the introduction of secondary misfit dislocations. The model emphasizes the determining role of the coalescence process in the formation of a dislocation network in heteroepitaxially grown $2 \mathrm{H}$-InN. Edge-type threading dislocations and dislocations of mixed character have been found to be dominating defects in the wurtzite InN layers. It has been shown that the threading dislocation density decreases exponentially during the film growth due to recombination and, hence, annihilation of dislocations, reaching $\sim 10^{9} \mathrm{~cm}^{-2}$ for $\sim 2200 \mathrm{~nm}$ thick InN films. () 2006 American Institute of Physics. [DOI: 10.1063/1.2363233]
\end{abstract}

\section{INTRODUCTION}

Recent studies of the narrow band gap InN compound semiconductor $(0.6-0.7 \mathrm{eV})$ show an increasing interest in investigating its fundamental properties and evaluate its potential applications. ${ }^{1-5} \mathrm{InN}$ was predicted to have the lowest effective mass for electrons among all III-nitride semiconductors, ${ }^{6}$ resulting in high mobility and high saturation velocity. The theoretical maximum mobility calculated for wurtzite $(2 H) \mathrm{InN}$ at $300 \mathrm{~K}$ is $\sim 4400 \mathrm{~cm}^{2} \mathrm{~V}^{-1} \mathrm{~s}^{-1}$, while at $77 \mathrm{~K}$ the limits are beyond $30000 \mathrm{~cm}^{2} \mathrm{~V}^{-1} \mathrm{~s}^{-1}$. It was found that $\mathrm{InN}$ exhibits an extremely high peak drift velocity at room temperature and achieves the highest steady-state peak drift velocity of $4.23 \times 10^{7} \mathrm{~cm} / \mathrm{s}$ among the III nitrides. ${ }^{8,9}$ It was also concluded that the transport characteristics of InN are superior to those of $\mathrm{GaN}$ and GaAs, over a wide range of temperatures from 150 to $500 \mathrm{~K}$ and doping concentrations up to $\sim 10^{19} \mathrm{~cm}^{-3}$. It was predicted that InNbased field-effect transistors (FETs) may have a cut-off frequency of over $1 \mathrm{THz}$ for $0.1 \mu \mathrm{m}$ gates. All the superior electronic properties of this material make InN a highly potential material for the fabrication of high-frequency heterojunction FETs. Another important discovery about wurtzite $\mathrm{InN}$ is its very strong surface charge accumulation on the order of $10^{13} \mathrm{~cm}^{-2}$. The accumulated electrons, which stay within $4 \mathrm{~nm}$ from the top InN surface, can be modulated by external treatments contributing considerably to the lateral

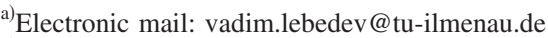

${ }^{b)}$ Also at Center for Micro- Nanotechnolgies of TU Ilmenau.
}

conductivity of thin InN films. Recently reported results reveal the promising applications of $\mathrm{InN}$ epilayers for chemical and biological sensors. ${ }^{10}$

The most valuable problem of $\mathrm{InN}$ heteroepitaxy is related to the large lattice mismatches between the epilayer and any available substrate material. In particular, due to the mismatch in the InN/ $\mathrm{Al}_{x} \mathrm{Ga}_{1-x} \mathrm{~N}$ heterosystem, the strain relaxation plays a crucial role in achieving high-quality heterostructures. The lattice mismatches in $2 \mathrm{H} \mathrm{InN/GaN} \mathrm{and}$ InN/AlN systems are -0.11 and -0.14 , respectively, and the disregistry of the interfacial atomic arrangements between the substrate and the epilayer will be accompanied by structural defects or by strain. This raises the question of strain relaxation even for very thin epilayers since the misfit dislocation (MD) density in InN is initially $>10^{11} \mathrm{~cm}^{-2} .{ }^{11}$ However, in order to be of a high optical or electronic quality, misfit relaxation defects should be avoided in these heterostructures as soon as possible within the constituting layers.

Extended defects, such as dislocations and stacking faults, are known to adversely affect III-nitride device properties causing nonradiative recombination, ${ }^{12,13}$ carrier scattering effects, ${ }^{14}$ and diffusion of dopants and impurities. ${ }^{15}$ Moreover, threading dislocation (TD) densities in the range of $10^{8}-10^{10} \mathrm{~cm}^{-2}$ arising as a result of enormous strains may ultimately limit III-nitride device performance. Thus, in the investigation of strain relaxation it is important to understand the structural, electronic, and optical properties of InN-based heterostructures. Strain evolution has been intensively studied for the growth of binary III nitrides $(\mathrm{GaN}$ and $\mathrm{AlN}){ }^{16-21}$ 
and their heterostructures, ${ }^{22-26}$ with a few studies being published on strain relief in $\mathrm{InN} / \mathrm{Al}_{x} \mathrm{Ga}_{1-x} \mathrm{~N}$ heterostructures. ${ }^{11,30-33}$ Most of the published contributions are focused on the measurement of the strain and strain relaxation with only a few reports upon the actual mechanisms involved in relaxation. ${ }^{16,27-29}$

The purpose of this article is to study those very mechanisms of strain relief, including the formation and elimination of dislocations, in wurtzite (0001) InN/AlN and $\mathrm{InN} / \mathrm{GaN}$ heterostructures grown by plasma induced molecular beam epitaxy (PIMBE). The type of misfit defects involved in strain relief at the interface between $\mathrm{InN}$ and the template on which it is deposited is characterized in detail for the case of AlN epitaxial templates. The relaxation of a lattice mismatch-induced strain is analyzed in situ during PIMBE by reflection high-energy electron diffraction (RHEED), ex situ by high resolution x-ray diffraction (XRD), and transmission electron microscopy (TEM) in both image and diffraction modes.

The following phenomena will be discussed in the framework of a model that emphasizes the decisive role of the coalescence process as revealed by RHEED, TEM, and XRD measurements: (1) plastic relaxation of the primary strain by the introduction of geometric misfit dislocations, (2) elastic strain relief during the island growth phase, (3) formation of threading dislocations induced by the coalescence of domains, (4) relaxation of elastic strain by the introduction of secondary misfit dislocations, and (5) and the mechanisms by which various dislocation slip systems are formed.

\section{EXPERIMENTAL DETAILS}

The samples were grown in a Balzer's PIMBE system with a base pressure of $\sim 1 \times 10^{-10}$ mbar. Standard Knudsen cells were used for $\mathrm{Ga}, \mathrm{Al}$, and In evaporations. An Oxford Applied Research MDR21 radio-frequency nitrogen plasma source has been used to supply active $\mathrm{N}$ radicals from purified $6 N 5 \mathrm{~N}_{2}$ gas. The substrate temperatures were calibrated by an infrared pyrometer. The growth process was monitored by digitized patterns of RHEED. Molecular fluxes were monitored in situ by a quadrupole mass spectrometer and calibrated using RHEED oscillations.

The samples consisted of epitaxial templates overgrown by a $2 H-\operatorname{InN}(0001)$ layer. The template structures and the epitaxial relationship in the heterosystems are summarized in Fig. 1. More details on the carbonization of Si surfaces ${ }^{34}$ and on the growth of $\mathrm{AlN}$ on $\mathrm{SiC} / \mathrm{Si}(111)$ (Ref. 35) and sapphire ${ }^{15}$ can be found elsewhere.

All template epilayers were grown in the twodimensional (2D) Frank-van der Merwe mode at substrate temperatures of $\sim 900$ and $\sim 710^{\circ} \mathrm{C}$ for $\mathrm{AlN}$ and $\mathrm{GaN}$, respectively. The III/N flux ratio was kept constant through the whole template epitaxy process to maintain slightly metalrich conditions for AlN and GaN growths. For all samples, an almost fully relaxed AlN layer was deposited as a buffer, typically $200-250 \mathrm{~nm}$ thick. In particular, XRD reciprocal space maps around the symmetric (0002) and the asymmetric $(20 \overline{2} 5)$ reflexes of $2 H$-AlN reveal a small increase of the
Four types of epitaxial templates investigated

\begin{tabular}{|c|c|c|c|}
\hline \multirow[b]{3}{*}{ Al-face } & & & Ga-face \\
\hline & Ga-face & Al-face & $\begin{array}{l}150 \mathrm{~nm} \\
\text { GaN }(0001)\end{array}$ \\
\hline & $\begin{array}{l}250 \mathrm{~nm} \\
\mathrm{GaN}(0001)\end{array}$ & $\begin{array}{l}250 \mathrm{~nm} \\
\mathrm{AlN}(0001)\end{array}$ & $\begin{array}{l}230 \mathrm{~nm} \\
\text { AlN(0001) }\end{array}$ \\
\hline $\begin{array}{l}200 \mathrm{~nm} \\
\Lambda \mathrm{NN}(0001)\end{array}$ & $\begin{array}{l}250 \mathrm{~nm} \\
\mathrm{AlN}(0001)\end{array}$ & $\begin{array}{l}4 \mathrm{~nm} \\
3 C-\mathrm{SiC}(111)\end{array}$ & $\begin{array}{l}4 \mathrm{~nm} \\
3 C-\mathrm{SiC}(111)\end{array}$ \\
\hline $\mathrm{Nl}_{2} \mathrm{O}_{3}(0001)$ & $\mathrm{Al}_{2} \mathrm{O}_{3}(0001)$ & $\operatorname{Si}(111)$ & $\operatorname{Si}(111)$ \\
\hline
\end{tabular}

$$
\begin{aligned}
& \text { Epitaxial relationships as detcrmincd by HRTEM } \\
& <01 \overline{1} 0>\mathrm{N}_{2} \mathrm{O}_{3} /<2 \overline{1} \overline{1} 0>\Lambda \mathrm{IN} /(<2 \overline{1} \overline{1} 0>\mathrm{GaN})<2 \overline{1} \overline{1} 0>\mathrm{InN} \\
& <2 \overline{1} \overline{1} 0>\mathrm{Al}_{2} \mathrm{O}_{3} /<01 \overline{\mathrm{T}} 0>\mathrm{AlN} /(<01 \overline{1} 0>\mathrm{GaN}) /<01 \overline{1} 0>\mathrm{InN} \\
& <0 \overline{1} 1>\mathrm{Si} /<0 \overline{1} 1>\mathrm{SiC} /<2 \overline{1} \overline{1} 0>\mathrm{AIN} /(\mathrm{GaN})<2 \overline{1} \overline{1} 0>\mathrm{InN} \\
& <001>\mathrm{Si} /<001>\mathrm{SiC} /<01 \overline{1} 0>\mathrm{AIN} /(\mathrm{GaN})<01 \overline{1} 0>\operatorname{InN}
\end{aligned}
$$

FIG. 1. The structure of the templates used in this work and the epitaxial relationship in the grown heterosystems.

in-plane stress (a decrease of the $c / a$ ratio) for the AlN layers grown on $\mathrm{Si}$ and sapphire. $c / a \sim 1.592$ for $2 \mathrm{H}$-AlN(0001)/ $\mathrm{Al}_{2} \mathrm{O}_{3}$ and $\sim 1.578$ for $2 \mathrm{H}-\mathrm{AlN}(0001) / 3 C-\mathrm{SiC} / \mathrm{Si}(111)$ have been deduced (nominal $c / a \sim 1.601$ ).

The templates were overgrown in situ by a $2 H$-InN epilayer at $T_{\text {sub }} \sim 380{ }^{\circ} \mathrm{C}$. The InN epilayers were grown under stoichiometric $(1: 1)$ conditions to prevent surface metal accumulation. The thickness of the InN layer has been varied from 0.35 to $2.2 \mu \mathrm{m}$.

TEM experiments were performed in two different microscopes: (i) TECNAI 20S-TWIN (FEI) and (ii) JEM-2011 (JEOL), both operating at $200 \mathrm{kV}$. Mechanical thinning and ion milling in a Gatan PIPS system were carried out in order to prepare the samples for cross-section (XTEM) and planview (PVTEM) inspections. Selected area electron diffraction (SAED) patterns and micrographs obtained in brightfield (BF) and dark-field (DF) modes by conventional twobeam (2B) conditions and high resolution TEM (HRTEM) were analyzed.

Structural analyses were performed by high resolution x-ray diffraction (HRXRD) using a Bruker D8 diffractometer. Rocking curves at the symmetric (0002) and reciprocal space maps at the symmetric (0002) and the asymmetric $(20 \overline{2} 5)$ InN reflections were taken to evaluate the crystal quality and the residual strain, respectively.

To study the surface properties, the samples were characterized in another ultrahigh vacuum (UHV) system with a base pressure of $\sim 1 \times 10^{-10}$ mbar described in more detail in Ref. 36. Due to the high reactivity of the InN surface the transfer to the analytical UHV chamber was performed with an absolute minimum time window of $30 \mathrm{~s}$. The surface morphology was measured in vacuo by atomic force microscopy (AFM) using contact mode (Fig. 2). A typical root mean square surface roughness of an $\mathrm{InN}$ epilayer having a thickness of $\sim 1 \mu \mathrm{m}$ does not exceed $2 \mathrm{~nm}$. The surface chemical composition and electronic properties (valence band) were studied by x-ray photoelectron spectroscopy (XPS) and ultraviolet photoelectron spectroscopy (UPS) using monochromated $\mathrm{Al} K \alpha(h \nu=1486.7 \mathrm{eV}), \mathrm{He}$ I $(h \nu=21.2 \mathrm{eV})$, or He II 


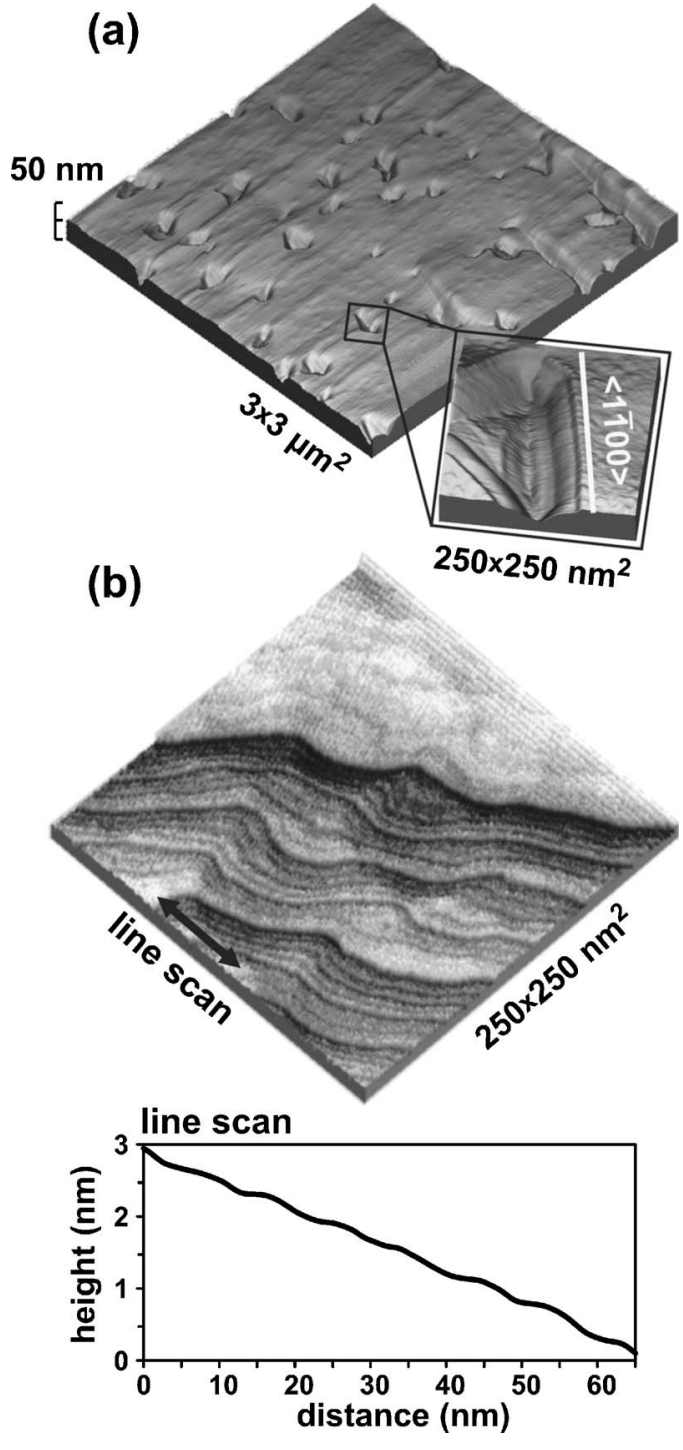

FIG. 2. (a) $3 \times 3 \mu \mathrm{m}^{2} \mathrm{AFM}$ scan of a $0.8 \mu \mathrm{m}$ thick InN epilayer grown on AlN $(0001)$ template. The inset shows a typical triangular hole created by the set of $(\overline{1} 100)$ and $(11 \overline{2} 0)$ planes of the islands due to the partial character of the coalescence phase. (b) $250 \times 250 \mathrm{~nm}^{2}$ AFM scan demonstrates the domain structure of an $\sim 30 \mathrm{~nm}$ thick $2 H$-InN nucleation layer. Bilayer-step morphology of the surface proves a $2 \mathrm{D}$ growth within nucleated islands.

$(h \nu=40.8 \mathrm{eV})$ radiation, respectively. All spectra are recorded using a hemispherical electron analyzer (EA125).

\section{STRUCTURAL AND SURFACE PROPERTIES OF $\ln N$}

The crystal quality of thick InN was analyzed by XRD. Rocking curves at the (0002) InN reflex show a homogeneous decrease of the full width at half maximum (FWHM) with the film thickness. The best FWHM of $0.277^{\circ}$ was found at about $1 \mu \mathrm{m}$ indicating the continuous improvement of the InN layers up to this thickness and a change in the relaxation mechanism thereafter. Thick InN layers of $\sim 1 \mu \mathrm{m}$ have only a small residual stress of $<0.1 \%$. These results agree very well with those previously reported for epitaxial InN layers grown at Cornell University. ${ }^{31}$ In particular, the dependence of $\Delta c / \Delta a$ is in excellent agreement, and thus the determined biaxial strain coefficient $R^{B}=2 C_{13} / C_{33}=$
TABLE I. Calculated residual stresses in the InN/AIN heterosystem during the nucleation and coalescence. $\mathfrak{I}_{x x}$ is the biaxial strain, $\mathfrak{I}_{z z}$ is the strain in the $z$ direction, $\sigma_{x x}$ is the biaxial stress $(\mathrm{GPa})$, and $\sigma_{z z}$ is the stress in the $z$ direction $(\mathrm{GPa})$.

\begin{tabular}{ccccc}
\hline \hline Method used & HRTEM & RHEED & RHEED & XRD \\
Growth phase & Nucleation & Nucleation & After coalescence & Averaged \\
\hline$\Im_{x x}$ & -0.0125 & -0.0411 & -0.0011 & -0.001 \\
$\Im_{z z}$ & 0.008 & 0.023 & 0.0006 & $\ldots$ \\
$\sigma_{x x}$ & -1.75 & -4.99 & -0.15 & $\ldots$ \\
$\sigma_{z z}$ & 0.65 & 1.85 & 0.05 & $\ldots$ \\
\hline \hline
\end{tabular}

$-\mathfrak{I}_{z z} / \mathfrak{I}_{x x}$ will be used to calculate the strain components $\mathfrak{I}_{x x}$ and $\mathfrak{I}_{z z}$ in plane and in growth direction, respectively (see Table I). For the calculation of $\mathfrak{I}_{z z}$ the experimental value of the biaxial strain coefficient $R^{B} \sim 0.6$ was used ${ }^{31}$ which differs from the most accepted theoretical value, $R^{B}=0.82$, derived using the elastic coefficients $c_{13}$ and $c_{33}$ provided by Wright. $^{37}$

The results of XPS and UPS measurements are presented in Fig. 3. After direct loading to the UHV system a small amount of oxygen and carbon contaminants were detected on the surface. From the XPS peak intensities a thickness of the contaminated layer, of $d \sim 0.5 \mathrm{~nm}$, was calculated. As conventional sputtering leads to the formation of metallic indium on the InN surface, ${ }^{36}$ the samples were annealed at $T$ $=500 \mathrm{~K}$ to reduce the amount of adsorbed molecules, resulting in $d \sim 0.1 \mathrm{~nm}$. AFM scans after heating showed that this procedure had no influence on the surface morphology, but the photoelectron spectra revealed some changes. The relevant core level peaks of the InN surface are shown in Fig. 3. All binding energies are given with respect to the Fermi level of a Ag reference sample. The binding energies (BEs) of the In $3 d_{5 / 2}$ and $\mathrm{N} 1 s$ are 443.9 and $396.2 \mathrm{eV}$, respectively. Upon annealing, changes in the chemical composition of the surface were observed. The $\mathrm{O} 1 s$ level consists of an adsorbate peak at $532.1 \mathrm{eV}$ and a small feature at $530.1 \mathrm{eV}$ originating from In-O bonds which is in good agreement with previous measurements. ${ }^{36}$ The oxygen is almost completely removed after annealing at $500 \mathrm{~K}$ (see Fig. 3). The amount of adsorbed carbon is only slightly affected by this procedure. The annealing leads to a change of the In $3 d_{5 / 2}$ signal. The shoulder at $\sim 445 \mathrm{eV}$ originating from the oxidized indium state is strongly reduced, matching the change in the $\mathrm{O} 1 s$ signal. The same behavior was observed for the In $4 d$ peak, also measured by UPS (He II), which has its maximum intensity at $17.8 \mathrm{eV}$. From both the In $3 d$ and In $4 d$ features, no formation of metallic indium was detected.

The N1s peak width is also reduced after heating. This can be explained by the removal of $\mathrm{NO}_{x}$ species at $\sim 397.5 \mathrm{eV}$ BE. From the UPS measurements the work function $\phi$ was obtained using the onset of secondary electrons when a bias of $-5 \mathrm{~V}$ was applied to the sample. The initial value for $\phi$ of $4.3 \mathrm{eV}$ changed to $4.1 \mathrm{eV}$ after annealing. The initial surface exhibits a structure at $5.3 \mathrm{eV}$ in the valence band which is most likely related to adsorbed species because after annealing this feature is removed and peaks at 6.8 and $3.1 \mathrm{eV}$ are observed (see Fig. 3). These structures origi- 


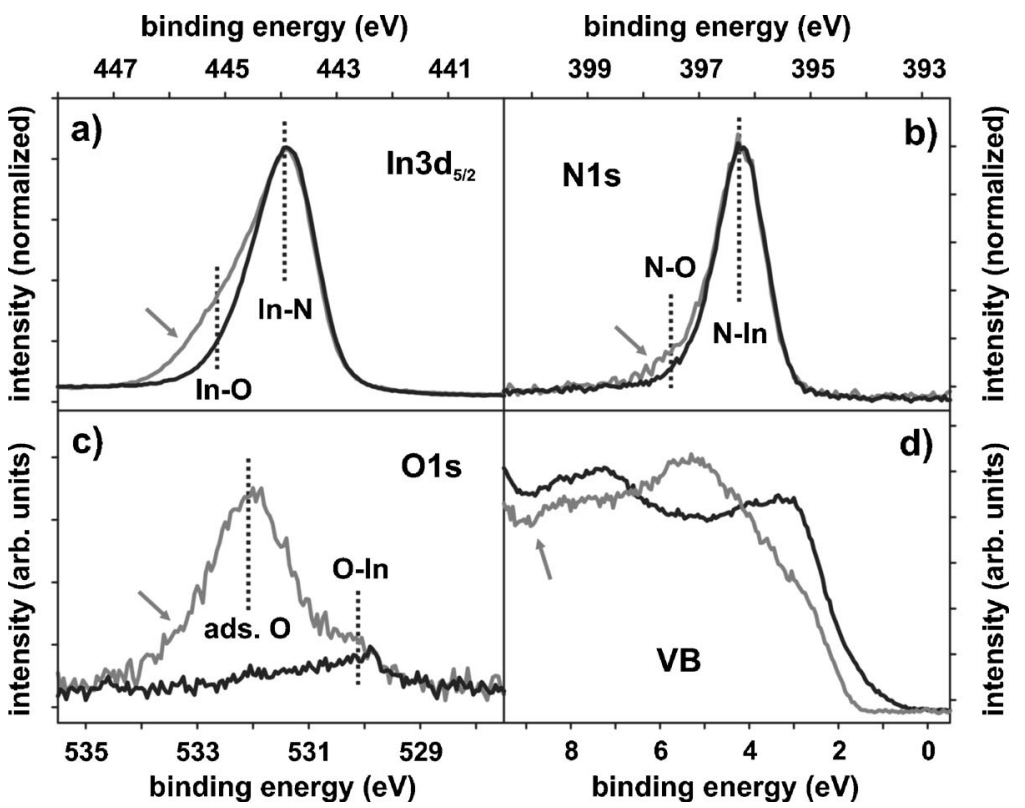

FIG. 3. Photoelectron spectra of the InN surface, [(a)(c)] In $3 d_{5 / 2}, \mathrm{~N} 1 s$, and $\mathrm{C} 1 s$ core levels measured using monochromated $\mathrm{Al} K \alpha$ radiation $(h \nu=1486.7 \mathrm{eV})$ and (d) valence band spectra measured using He II radiation $h \nu=40.8 \mathrm{eV}$. The spectra marked by arrows correspond to the as loaded sample, in comparison to the surface after annealing at $500 \mathrm{~K}$. nate from nitrogen electron states in indium nitride and have been previously observed experimentally ${ }^{38}$ and predicted by theoretical calculations. ${ }^{39}$ The valence band (VB) maximum at the surface is found to be $1.6 \mathrm{eV}$ below the Fermi edge. This strong downward band bending can be explained by the observed high electron concentration of $\mathrm{InN}$ surfaces.

\section{GROWTH DYNAMICS}

In all the experiments, the growth of InN (0001) was initiated in situ on the epitaxial template [i.e., (0001) AlN or $\mathrm{GaN}$, see Figs. 4(a) and 4(b) at $380^{\circ} \mathrm{C}$ ]. The growth parameters were not varied during the process and similar growth conditions were used for PIMBE of InN on both AIN and GaN templates.

Identical changes observed in the RHEED patterns during the growth indicate that in both cases (i.e., AlN and GaN templates) the film growth is affected by similar mechanisms. The only difference was observed in the characteristic relaxation time (thickness) of the in-plane lattice parameter. Moreover, differences in electron transport properties of InN epilayers grown on both $\mathrm{AlN}$ and GaN templates were not observed. Thus, in the following sections, we restrict our considerations to the case of $\operatorname{InN}(0001) / \mathrm{AlN}(0001)$ heterosystem, which, due to the larger lattice misfit, is more illustrative to show the relaxation phenomena.

The in situ RHEED observations allowed us to distinguish at least three different growth phases characteristic for any highly mismatched heterosystem. These stages are schematically shown in Fig. 4(c). (1) (A) Three-dimensional (3D) nucleation and subsequent growth of slightly misoriented $2 \mathrm{H}$-InN islands up to a thickness of $\sim 10-15 \mathrm{~nm}$ (a pure Volmer-Weber mode), (2) (B) a partial coalescence of the islands into a continuous film, and (3) (C) a long-term epitaxy of $2 H$-InN followed by the gradual improvements of the surface morphology.

To analyze the growth dynamics, we consider a classic growth model applied in our earlier work for the case of $2 H$-AlN/3C-SiC/Si(111) growth. ${ }^{35}$ In the simplest case, the growth mode is determined by the balance between the formation energies of the film surface $\sigma_{\text {film }}$, the template surface $\sigma_{\text {sub }}$, and the interfacial energy $E_{\text {int }}$. One can write the conditions for the 2D growth of a strained thin film as

$$
\sigma_{\text {sub }}>\left(\sigma_{\text {film }}-\Delta E_{M}\right)+E_{\text {int }}-\Delta \mu / 2 A_{(h k l)}+n_{\text {lat }} E_{\text {str }},
$$

where $n_{\text {lat }}=3$ for the hexagonal cell, $E_{\text {str }}$ is the macroscopic stress energy, $A_{(h k l)}$ is an intersection of the elemental cell
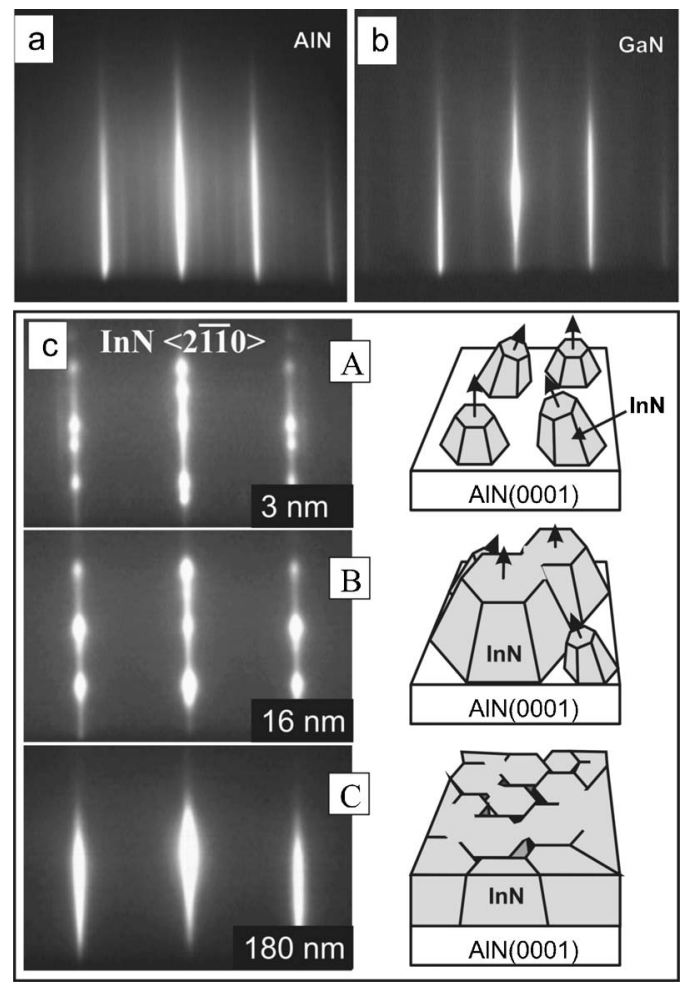

FIG. 4. $\langle 2 \overline{1} \overline{1} 0\rangle$ RHEED patterns of (a) a $200 \mathrm{~nm}$ thick $\operatorname{AlN}(0001)$ and (b) $220 \mathrm{~nm}$ thick $\mathrm{GaN}(0001)$ epilayers. (c) Schematic representation of the growth of $2 \mathrm{H}$-InN on highly mismatched templates: (A) early formation of the slightly misoriented flat $\mathrm{InN}$ platelets with elastic relaxation at the edges, (B) partial coalescence of the platelets, and (C) further growth and formation of a continuous layer. 
with the surface plane $(h k l)$, and $\Delta E_{M}$ states for the reduction in the total energy of the system due to the periodical undulations at the heterointerface. ${ }^{40}$

The two last terms in the Eq. (1) represent the formation energy of the epilayer. $\Delta \mu=\mu-\mu_{\text {bulk }}=k_{B} T \ln (S)$ is the difference in the chemical potentials of the bulk and gas phases of the species representing the actual growth conditions being a function of the supersaturation $S$ and temperature $T$. The actual growth conditions can be described by the following relation:

$$
\mu_{\text {In }}=\mu_{\text {bulk }}^{\mathrm{InN}}-\mu_{\text {bulk }}^{\mathrm{N}}+\Delta H_{f}^{\mathrm{InN}}+\Delta \mu_{\text {In }},
$$

where $\Delta H_{f}^{\mathrm{InN}}$ is the heat of formation of the binary compound. The conditions $\Delta \mu_{\mathrm{In}}=-\Delta H_{f}^{\mathrm{InN}}$ and $\Delta \mu_{\mathrm{In}}=0$ imply $\mathrm{N}$-rich and In-rich growths, respectively.

As it follows from Eq. (1), the supersaturation is the only parameter capable of changing the growth mode at the nucleation stage. At the flat surface and low supersaturation used for the stoichiometric growth, the term $\Delta \mu / 2 A_{(h k l)}$ does not affect the overall energy balance. As a result, the stoichiometric nucleation and growth have a pure 3D character due to an enormous value of $E_{\mathrm{str}}$, until the coalescence phase. The use of a higher supersaturation for the nucleation can shift the energy balance towards the Stranski-Krastanov growth mode. In particular, predeposition of 2-3 ML (monolayer) of In on the flat $2 \mathrm{H}$-AlN surface before the epitaxy results in the formation of a very thin $2 \mathrm{D}$ wetting layer of $\mathrm{InN}$, due to the presence of activated nitrogen in the chamber volume. However, the experimentally observed characteristic transition into 3D growth takes place after deposition of 1-2 $\mathrm{ML}$ of InN. Consequently, as InN growth conditions remain constant, the last term in Eq. (1), $E_{\text {str }}$, plays the dominant role in growth dynamics controlling the mechanisms involved in the lattice relaxation.

For a particular bicrystal consisting of a semi-infinite substrate and an epilayer of thickness $d$, the energy per unit area $E_{\mathrm{el}}$ of a grid of edge dislocations having Burger's vectors $\mathbf{b}$ laying on the growth plane, with dislocation spacing $p$ is given approximately by

$$
E_{\mathrm{el}}=n_{\mathrm{lat}} \frac{G|b|^{2}}{4 \pi(1-\nu) p} \ln \left(\frac{e d}{r_{0}}\right) .
$$

The elastic strain remaining is given by $\mathfrak{I}=f-|\mathbf{b}| / p$. The total strain energy per unit area in Eq. (1), $E_{\text {str }}$, is then given by

$$
E_{\text {str }}=n_{\text {lat }} \frac{G(1+\nu)}{4 \pi(1-\nu) p} \Im^{2} d+n_{\text {lat }} \frac{G|b|(f-\Im)}{2 \pi(1-\nu)} \ln \left(\frac{e d}{r_{0}}\right),
$$

where $G$ and $\nu$ are the interfacial shear modulus and Poisson's ratio of the epilayer, respectively, $\mathfrak{I}$ is the biaxial stress remaining in the epilayer, and $r_{0}$ is the inner core radius of dislocation. $^{41-43}$

In Eq. (4), the first term represents the elastic strain energy, which is the driving force for the relaxation of the lattice during growth. The second term is the energy accumulated by the dislocations network and corresponds to the plastic relaxation associated with dislocation formation.

In our experiments, the in-plane lattice relaxation was
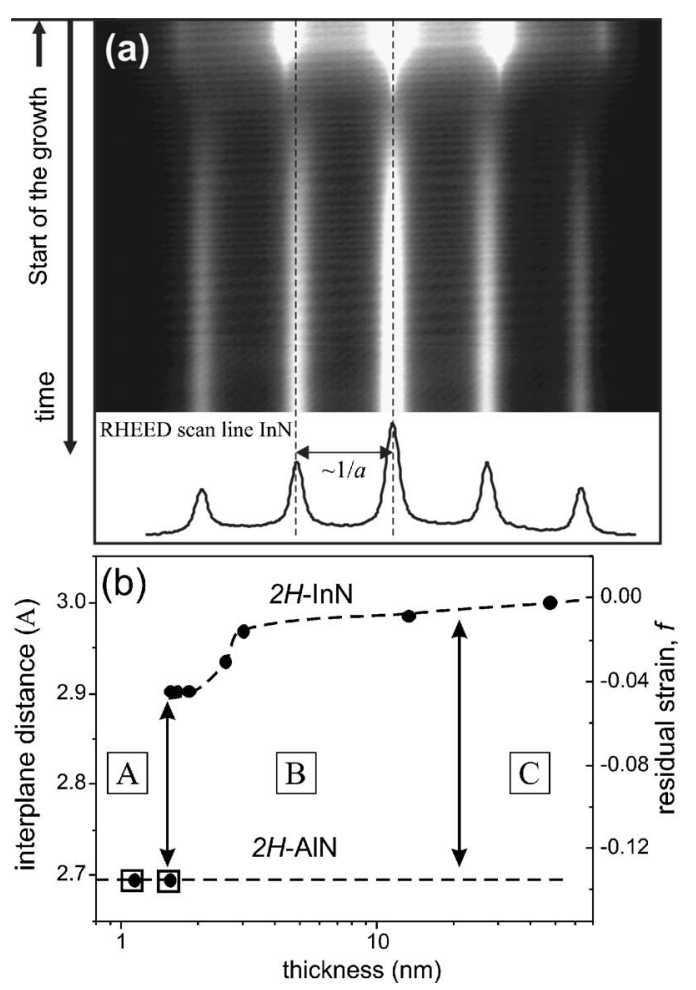

FIG. 5. In-plane lattice parameter evolution during the nucleation and growth phases of InN on AIN template. (a) A bitmap image presents a time dependence of $\langle 2 \overline{1} \overline{1} 0\rangle$ RHEED intensity profiles. The height of the image equals $90 \mathrm{~s}$ of growth time. (b) In-plane lattice parameter extracted by the profile analyses vs thickness of the InN layer.

monitored in situ by recording the intensity profile of RHEED patterns. A bitmap image, shown in Fig. 5(a), presents a time dependence of the RHEED intensity profile during the nucleation and coalescence phases of the InN growth. Every horizontal line (1 pixel thick) in the image represents the intensity profile averaged over $1 \mathrm{~s}$ of the growth. The height of the image equals $90 \mathrm{~s}$ of growth time. The lateral resolution of 752 lines per $6 / a$ corresponds to the real space resolution of $\sim 0.02 \AA /$ pixel. The corresponding variations of the in-plane lattice parameter were derived from the continuous variations of the spacing between planes of the type $\{01 \overline{1} 0\}$ perpendicular to the basal plane. It is presented in Fig. 5(b) as a function of the epilayer thickness showing the general trends of the misfit accommodation between AlN template and InN epilayer. As one can see, the different stages of the relaxation of the InN in-line lattice parameter correspond well to the three growth phases shown in Fig. 4(c). While the phases $A$ and $B$ are characteristic by the 3D growth mode, which is typical for highly stressed epilayers, phase $C$ typically occurs in the 2D mode resulting in a slow relaxation rate and in a smooth epilayer surface [Fig. 6(a)]. Moreover, it is obvious from the order of the variation in the lattice parameter that both plastic as well as elastic mechanisms are involved in the primary misfit accommodation between InN epilayers and $2 \mathrm{H}$-AlN templates. The biaxial strain due to the thermal mismatch arising during the cooling of the films is out of the scope of this work. It should be mentioned that the major portion of this stress arises at temperatures below the brittle-ductile transition temperature of 

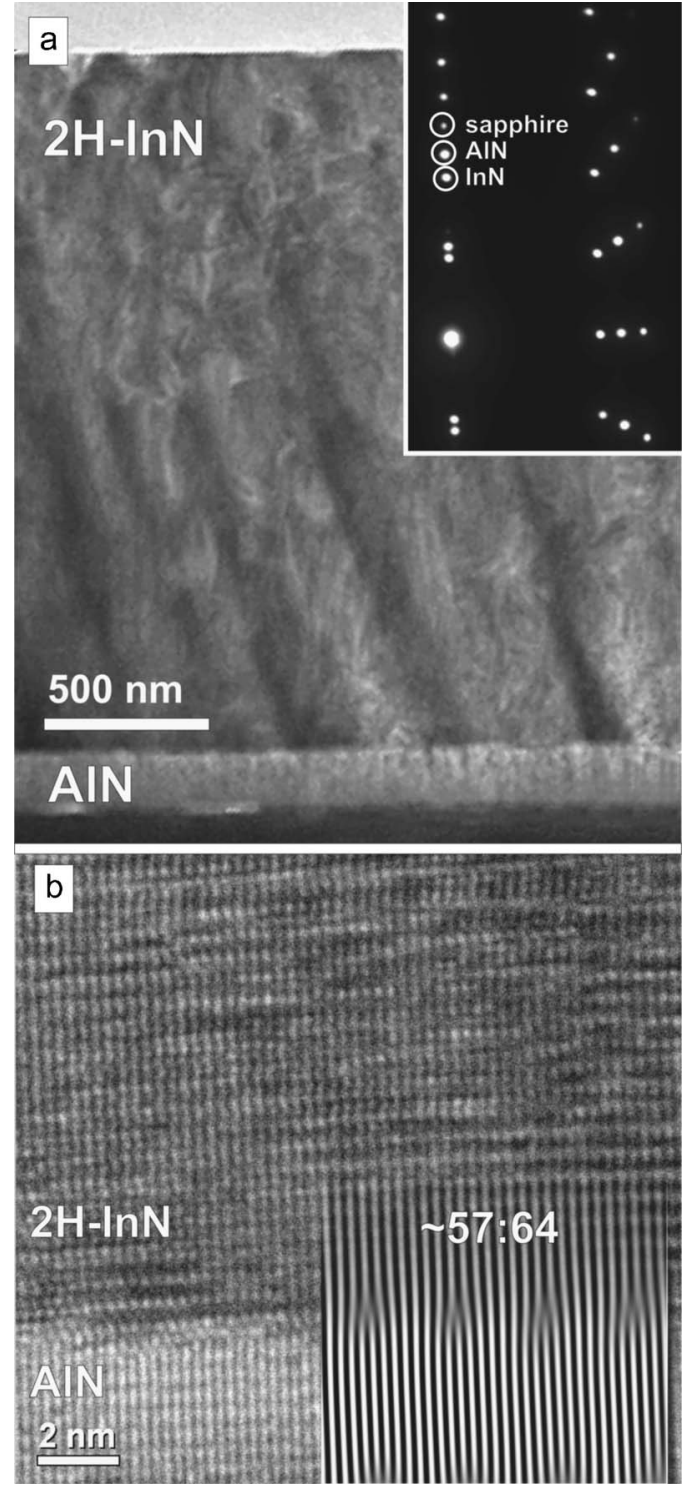

FIG. 6. Epitaxial relationship and atomic-plane relationship at the AlN/InN heterointerfaces: (a) Bright-field XTEM micrograph of a $2.2 \mu \mathrm{m} 2 \mathrm{H}$-InN layer grown on an $\mathrm{AlN} / \mathrm{Al}_{2} \mathrm{O}_{3}$ template (general view); inset: $\langle 2 \overline{1} \overline{1} 0\rangle \mathrm{SAED}$ pattern showing sapphire, AIN and $\mathrm{InN}$ related aligned reflection spots; the following epitaxial relationships were observed: $\langle 01 \overline{1} 0\rangle \mathrm{Al}_{2} \mathrm{O}_{3} /\langle 2 \overline{1} \overline{1} 0\rangle \mathrm{AlN} /$ $\langle 2 \overline{1} \overline{1} 0\rangle \mathrm{InN}$ and $\langle 0001\rangle \mathrm{Al}_{2} \mathrm{O}_{3} /\langle 0001\rangle \mathrm{AlN} /\langle 0001\rangle \mathrm{InN}$. (b) HRTEM image in the $\langle 2 \overline{1} \overline{1} 0\rangle$ zone axis; inset: The fast Fourier filtered HRTEM image shows the $(01 \overline{10})$ net planes of AlN and InN demonstrating the match between template and epilayer.

$2 H$-InN. As a result, some cracks may be introduced into the film.

\section{LATTICE RELAXATION IN $2 H-I n N$}

It was generally found that the initial relaxation in the highly mismatched wurtzite III-nitride heterosystem occurred via a three-step growth mechanism consisting of the following: the formation of planar platelets, their partial coalescence, and, finally, the introduction of dislocations, most likely at the edges of the platelets. ${ }^{16,24,30,44,45}$ A similar model was used successfully to explain 2D PIMBE of $2 H$-AlN on $\mathrm{Si}(111)$ (Ref. 46) and (001) (Ref. 47) substrates. It is also

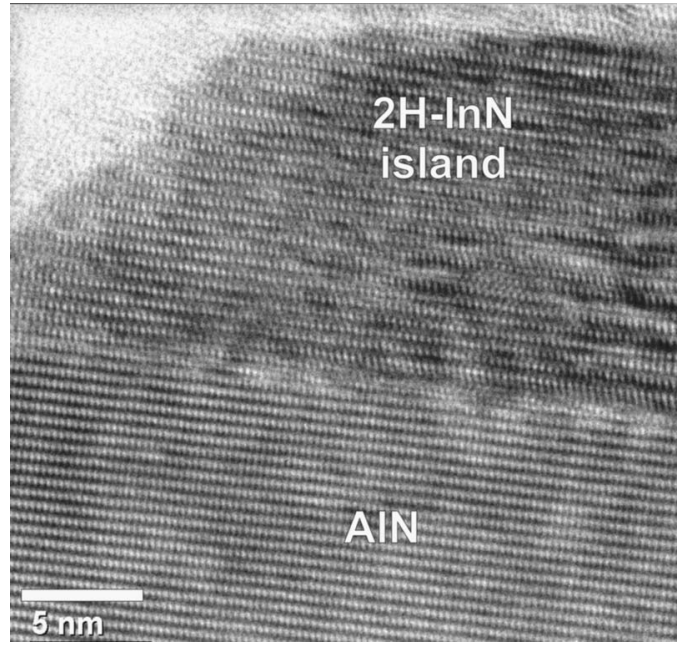

FIG. 7. HRTEM image of a $2 H$-InN island nucleated on an AlN template.

important to note that, in the case of $2 \mathrm{H}-\mathrm{InN} / \mathrm{Al}_{x} \mathrm{Ga}_{1-x} \mathrm{~N}$ heterosystems, all the steps presume different mechanisms of strain relaxation.

The growth of $2 \mathrm{H}$-InN involves deposition of a film, which is lattice mismatched with respect to any available template. Assuming two perfectly rigid relaxed crystals, the film/template misfit parameter $f$ is equal to -0.11 and -0.14 for $\mathrm{InN} / \mathrm{GaN}$ and $\mathrm{InN} / \mathrm{AlN}$ heterosystems, respectively. Such systems cannot be grown pseudomorphically. ${ }^{48}$

In systems with large misfit parameters (depending on the elastic moduli of the film, $f>4 \%-5 \%$ ) most of the lattice mismatch is accommodated by misfit dislocations (MDs), which are physically distinct from the classical van der Merwe-Matthews-type MDs. ${ }^{49}$ In $\mathrm{InN} / \mathrm{Al}_{x} \mathrm{Ga}_{1-x} \mathrm{~N}$, one may observe an approximate matching of $m\{01 \overline{1} 0\}_{\text {InN }}$ planes of the film with $n\{01 \overline{1} 0\}_{\text {template }}$ planes of the template. Extra half planes define geometrical misfit dislocations (GMDs), $60^{\circ}$ dislocations with $\mathbf{b}$ and line parallel to $\langle 2 \overline{1} \overline{1} 0\rangle$ directions and lying on the (0001) basal plane, which exist in a high percentage from the very start of the film growth [Fig. 6(b)].

Besides the accommodation of the initial misfit by GMDs, there is another way to relax misfit strain destabilizing the growth front by producing surface modulation or eventually forming islands. Moreover, on the planar top surface of each platelet, the layer-by-layer growth mode can be achieved if the conditions in Eq. (1) are satisfied, similar to what has been observed for AlN/GaN and GaN/AlN $2 H$ heterosystems. ${ }^{16}$ The corresponding term in Eq. (1), $\Delta E_{M}$, describes the reduction in the total energy of the system due to periodic undulations at the growing surface. ${ }^{39}$

It was also reported ${ }^{16,44}$ that the nucleation step results in a second-order elastic relaxation of the epilayer within the nucleated nanoplatelets of slightly different orientations [see Figs. 4(c) and 5(b), phase $A$ ]. In the case of the InN/AlN(0001) heterosystem, their lateral sizes reach diameters of $\sim 50 \mathrm{~nm}$ with heights of $10-15 \mathrm{~nm}$ before the coalescence takes place (see Fig. 7). As we will discuss later on, due to observed relaxation, the residual biaxial strain in the nanoplatelets is not large enough to introduce dislocations into the growing islands on the stage of $3 \mathrm{D}$ growth. Addi- 
tionally, it has been shown by Cantu et al. ${ }^{50}$ by means of TEM and XRD analysis that inclined edge TDs also contribute to the initial relaxation process by a TD line redirection at the interface. In our particular case, TEM investigations indicate that the TDs penetrating from the template into the InN layer are indeed inclined providing a MD component (see Fig. 8).

The next growth step, the partial coalescence of islands [see Figs. 4(c) and 5(b), phase $B$ ], leads to the formation of a high density of either phase boundaries (PBs) [Fig. 8(a)] (in the case of the occasional existence of a metastable $3 C$ InN polytype) or domain boundaries (DBs) [Figs. 8(b) and $8(\mathrm{c})]$. The efficiency of the coalescence and the surface smoothing depend critically on the stoichiometry of the deposited species and therefore affected by the actual supersaturation. The partial coalescence may cause an additional undesirable effect: the immobilization of incipient dislocations generated from lattice misfit strain relaxation within pinholes between islands. This dislocation "trapping" effect can inhibit dislocation annihilations [see Fig. 8(d)], which requires dislocation gliding. ${ }^{44}$ In general, the partial character of the coalescence presumes that $2 \mathrm{H}$-InN epilayers have a pronounced domain structure causing variations in the crystal quality as well as in the carrier transport properties of the film.

The coalescence is followed by the gradual introduction of secondary misfit dislocations (SMDs) due to the rising biaxial stress energy in the continuous epilayer. These SMD segments with lines propagating parallel to the interface have associated dislocation portions threading towards the surface, namely, "secondary" TDs, whose density changes with the thickness and will be designated in a later section as $D_{2}(d)$. Note that the SMD portions will be able to relieve a major component of the actual thickness-dependent residual stress only by the glide of the secondary TD in the most favorable slip systems (Matthews-Blakeslee mechanism). ${ }^{33}$ This mechanism can play a dominant role, if $2 \mathrm{D}$ growth conditions (layer by layer or step flow growth) are reached by an appropriate choice of In supersaturation and/or substrate temperature. $^{15}$

In general, depending on the sign and the order of the remaining strain, other mechanisms could participate to relax biaxial strain in the epilayer, e.g., the propagation of cracks (tensile stress) or film decohesion (compressive stress). ${ }^{16}$ All mechanisms mentioned above are of high importance for epitaxy of high-quality InN layers and will be discussed in the following sections.

\section{DISCUSSION}

\section{A. Plastic relaxation by geometrical misfit dislocations}

Shown in Fig. 6, TEM and HRTEM images demonstrate a cross-sectional overview, and an atomic-plane relationship at the AlN/InN heterointerface. Fast Fourier filtered HRTEM images (see inset) demonstrate the fit between the $\{01 \overline{1} 0\}$ net planes of InN and AlN displayed along the $\langle 2 \overline{1} \overline{1} 0\rangle$ direction. Note that similar relationships were found in the $\mathrm{InN} / \mathrm{GaN}$ interface. The derived approximate ratio, $m: n$, for

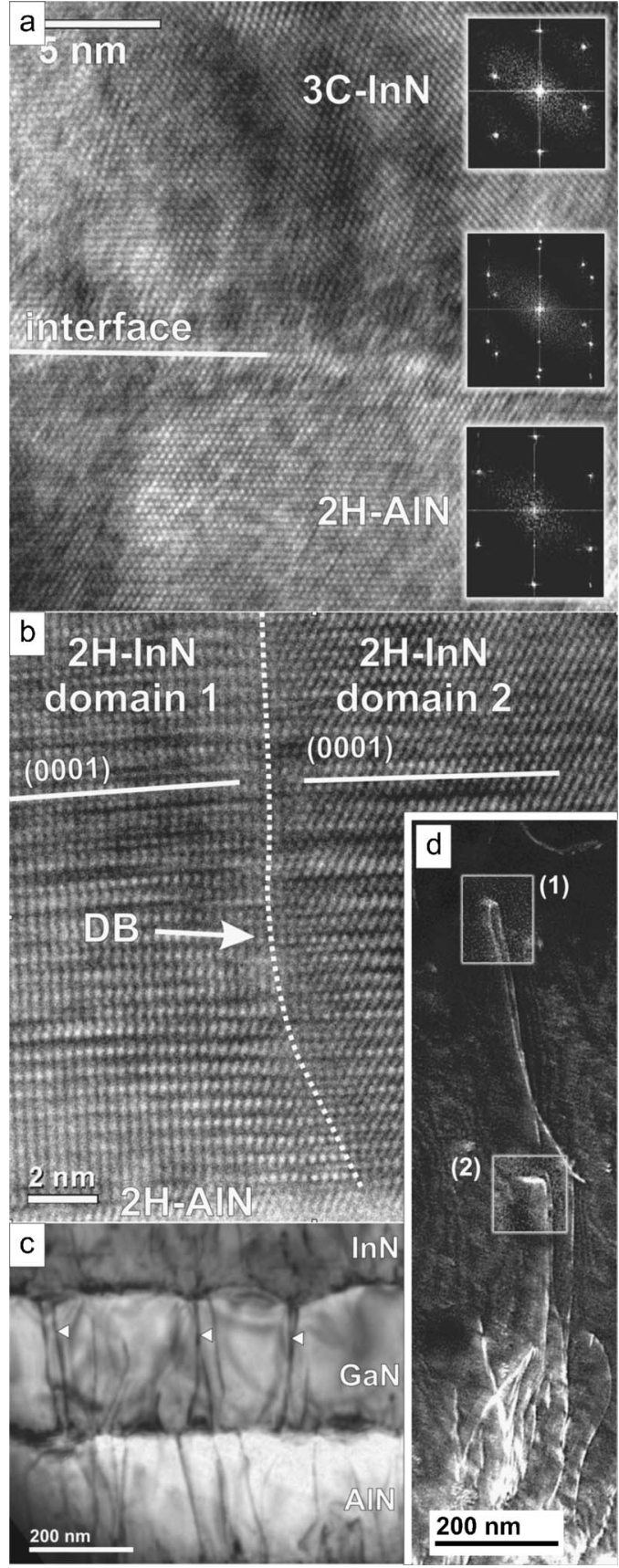

FIG. 8. TEM micrographs of typical phases, boundaries, and dislocations types in $\langle 2 \overline{1} \overline{1} 0\rangle \mathrm{AlN} /(\mathrm{GaN}) / \mathrm{InN}$ heterosystems. (a) $3 C$-InN metastable phase inclusion along the $\langle 011\rangle$ zone axis placed on AIN [insets: fast Fourier transform (FFT) spectra of InN (top) InN/AlN heterointerface (middle) and InN (bottom) are similar to the corresponding SAED patterns and demonstrate the cubic nature of $3 C$-InN as well as the hexagonal crystallography of $2 \mathrm{H}$-AlN]. (b) HRTEM image of a $2 \mathrm{D}$ DB between two tilted $2 \mathrm{H}$-InN domains placed on AlN template; (c) 2D DB network in a $2 \mathrm{H}$-GaN epilayer between AlN and InN structures, after the partial coalescence of domains (triangles point out to 2D boundaries). (d) Dark-field (DF), two-beam (2B) conditions using the 0002 reflection allow us to image a TD network in the bulk of hexagonal InN grown on AlN. The density of TDs decreases as the layer becomes thicker: (1) and (2) exemplify TD trapping and/or annihilation.

the $\{01 \overline{1} 0\}$ planes InN/AlN is 57:64, as followed from the statistical HRTEM analyses. Consequently, the primary mismatch, mainly accommodated by a series of GMDs, is $\left|\left(n \cdot d_{\mathrm{AlN}}-m \cdot d_{\mathrm{InN}}\right)\right| / n \cdot d_{\mathrm{AlN}}=-0.013$ for InN/AlN. Thus, the 
line density of dislocations in $\mathrm{InN}$ near the AlN/InN heterointerface extracted from HRTEM images is $D_{\mathrm{I}} \sim(4 \pm 0.5)$ $\times 10^{6} \mathrm{~cm}^{-1}$. The introduction of MDs parallel to $\langle 11 \overline{2} 0\rangle$ accommodates the main part of the mismatch $(\sim 12.2$ from the total $\sim 13.5 \%)$ in the InN/AlN heterosystem. We propose that those MDs detected by HRTEM in $\langle 2 \overline{1} \overline{1} 0\rangle$ directions should be mainly GMDs plus a minor contribution of the SMDs (with a line parallel to the $\langle 2 \overline{1} \overline{1} 0\rangle$ direction and $\mathbf{b}$ $=1 / 3\langle 11 \overline{2} 3\rangle)$ formed for relaxing the biaxial strain at the heterointerface. ${ }^{33}$ The very regular spacing between misfit dislocations only could be explained by a plastic relaxation through GMD generation, since the reaccommodation of misfit dislocations by slip in the basal plane is not very probable.

The corresponding values of the calculated residual stress at different growth stages measured by different methods are listed in Table I. Here, Refs. 51 and 52 have been used as a source for InN elastic constants and stress calculation formalism, respectively. As we commented, the plastic relaxation degree measured dynamically by RHEED is smaller than those derived from HRTEM. Three possible reasons can explain this lack of correspondence. (i) The atomicplane ratio varies along the interface being a function of the local growth conditions; (ii) the initial ratio has been changed due to the recrystallization of the domains during coalescence or postcoalescence phase; and (iii) either additional dislocations or cracks form in the epilayer due to the biaxial strain induced by the thermal mismatch arising during the cooling of the sample.

On the other hand, the RHEED observations represent the data integrated over, at least, $3 \mathrm{~mm}^{2} \mathrm{InN}$ surface and can be used as an averaged value. In both cases, however, the magnitude of the residual misfit is small enough to allow the continuation of the second-order elastic relaxation of the epilayer within the nucleated nanoplatelets. Moreover, the influence of the nucleation phase on the electrical and transport properties of the thick $(>400-500 \mathrm{~nm}) \mathrm{InN}$ film is not significant. As we will discuss later on, the quality of the InN epilayer is determined mainly by the coalescence.

\section{B. Elastic relaxation through island growth}

After the primary relaxation of the InN domains via introduction of GMDs, but before a plastic release of the strain energy via formation of a threading dislocation network, an elastic relaxation of islands takes place. Approximated analytical solutions for the equilibrium stresses and strains have been discussed in the literature for a periodic set of thin deposites. ${ }^{16,51}$ It was shown that the two facing crystals no longer share the natural misfit, but share a so-called active misfit involving the surface stresses of the substrate. This surface stress may enhance, compensate, or decrease the epitaxial misfit. Moreover, the island relaxes by its free borders so that the lateral stress becomes height dependent.

In the frame of a 2D thick ribbon model suggested by Kern and Müller, ${ }^{51}$ a simple isotropic approximation for the relaxation of the topmost layers of the $2 \mathrm{H}$-InN islands can be

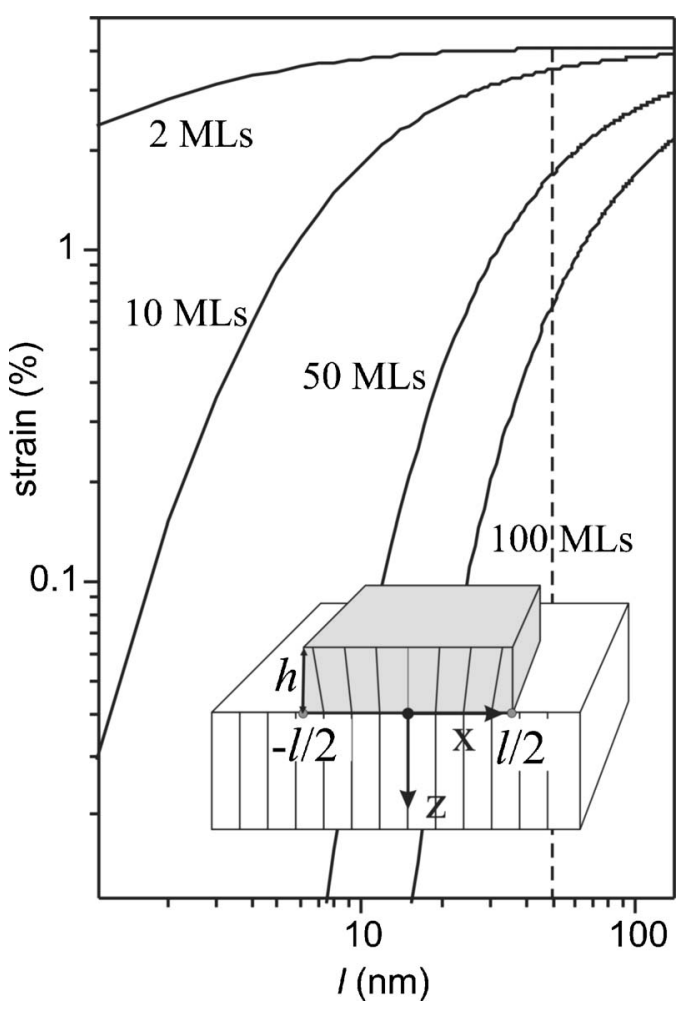

FIG. 9. Calculated in-plane strain due to the island growth as a function of characteristic island size $l$ for different thicknesses.

obtained. The model assumes that the strain in an infinitely long ribbon of height $h$ and width $l$, deposited coherently on a substrate having a misfit $f_{0}$, is given by

$$
\begin{aligned}
& \mathfrak{I}_{y y}(x, N)=\mathfrak{I}_{y y}(x, 0)\left\langle M_{1}\right\rangle^{N-1}, \\
& \left\langle M_{1}\right\rangle=1-\frac{2 \pi a}{l}+\left(1+\sqrt{\frac{2 \pi a}{l}}\right)^{2} \exp \left(-\sqrt{\frac{2 l}{a \pi}}\right),
\end{aligned}
$$

where $N$ defines the number of monolayers and varies from 0 to $h / a$ - the total number of monolayers included in the ribbon of height $h$.

Based on these approximations, we can interpret the experimental results obtained by AFM and in situ RHEED analyses to calculate a strain relief provided by the $3 \mathrm{D}$ growth. Assuming a rigid substrate with $f_{0}=-0.0411$ (primary misfit is partly relaxed by GMDs) and a typical island size of $\sim 50 \mathrm{~nm}$ [see Figs. 1(b) and 8], which is large compared to atomic distances; an approximate value of $\sim 4 \%$ for the strain relief through the island formation can be obtained. This value fits well to the RHEED observations [see Fig. 5 (b), phase $B$ ] showing similar changes in the in-plane lattice parameter during the $3 \mathrm{D}$ growth phase. The elastic strain relief occurring within the island can be reversible ${ }^{16}$ or might be compensated during the coalescence. However, the observed relaxation has an obvious consequence for the growth. It prevents the formation of a dislocation network inside the growing islands.

The residual strain in $2 \mathrm{H}$-InN determines the characteristic size of the growing islands, preventing the growth of large area domains during the nucleation stage. As one can see in Fig. 9, for thin platelets ( $\sim 10 \mathrm{ML})$, the onset of sig- 
nificant strain reduction starts at $\sim 50 \mathrm{~nm}$. This value corresponds to the experimentally observed characteristic size of the InN islands taking part in the subsequent coalescence.

Two important notes concerning the model restrictions have to be made. The model does not take into account a plastic deformation of the islands as well as kinetic parameters governing platelet growth. ${ }^{16}$ Obviously, the relaxation may contain both elastic and inelastic components, and the shapes of the islands play an important role and can prevail over the elastic effects. Furthermore, since surface stresses are adsorption sensitive, a surface adsorption may force the equilibrium strains and stresses to change in a reversible fashion.

\section{Dislocation slip systems in $\mathbf{2 H}-\mathrm{InN}$}

The density of GMDs is quite high reaching values of $D_{\text {I }} \sim 4 \times 10^{6} \mathrm{~cm}^{-1}$ as determined by HRTEM. However, the area of high degree lattice distortions is restricted by the near interface region (of order of $2-3 \mathrm{~nm}$ ) accommodating the primary misfit and increasing the interfacial energy. ${ }^{46}$

Besides the misfit dislocations at the interface, different types of dislocations are formed in the volume of $2 \mathrm{H}$-InN epilayers by a number of mechanisms including (a) growth errors (e.g., point and basal plane defects), (b) propagation of TDs from the underlying epitaxial AlN template $\left(D_{t} \approx 4\right.$ $\times 10^{9} \mathrm{~cm}^{-2}$ ), which could be bent from the growth direction to the interface plane, (c) partial coalescence of the adjacent domains [ TDs and DBs, see Figs. 8(b)-8(d)], (d) polytype transition [introduction of $3 C$-phase grains with PBs, see Fig. 8(a)], (e) residual and thermal mismatch stresses accommodating the elastic strains, etc. The latter types of dislocations (typically van der Merwe-Matthews type) are generally produced by segments of MDs that lie at or parallel to the interface and are connected to TDs in the film or the template. $^{49}$ These segments are higher in number as the biaxial strain (actual/local residual strain) increases, so their local densities are lower as the thickness increases, although their accumulated/absolute number increases with thickness. According to XRD measurements, the grown layers are almost fully relaxed after $350 \mathrm{~nm}$ of growth. Thus, these segments should not continue appearing after this level and they should start after the coalescence process.

Dislocations in the wurtzite III-nitride structure can be defined by their slip systems. ${ }^{33,49,52}$ In the order of increasing importance (from the strain relief and occurrence point of view), it can be defined as (a) basal dislocations [i.e., those lying on the (0001) plane] with $\mathbf{b}=1 / 3\langle 11 \overline{2} 0\rangle$, (b) prismplane dislocations [i.e., (i) those lying on a $\{1 \overline{1} 00\}$ plane] with $\mathbf{b}=1 / 3\langle 11 \overline{2} 0\rangle$ (pure edge) or $\mathbf{b}=\langle 0001\rangle$ (pure screw), and (ii) those lying on a $\{11 \overline{2} 0\}$ plane, and (c) pyramidalplane dislocations [i.e., (i) those lying on a (10 $\overline{1} 1)$ plane] with $\mathbf{b}=1 / 3\langle 11 \overline{2} \overline{3}\rangle$; (ii) those lying on $\{1 \overline{1} 01\}$ with $\mathbf{b}$ $=1 / 3\langle 11 \overline{2} 3\rangle$; and those lying on $\{1 \overline{10} 2\}$ planes.

Logically, the movement of dislocations lines tends to happen along the more atomically closed-packed planes of the wurtzite system, but the order of importance is reversed [from (a) to (c)] with respect to the surface atomic density.
This fact has a clear explanation: as it follows from Eq. (3), the activation of any stress-induced dislocation system depends on the occurrence of a sufficiently large resolved shear stress on that particular slip system (i.e., on the slip plane along the Burger's vector). In general, systems that have the highest resolved shear stress are activated first. In the case of $2 \mathrm{H}$-InN, it is difficult to estimate the magnitude and direction of the resolved shear stress on the different systems because very little is known about the state of the stress in the film. However, it is well known that any type of stress generated in the film because of film/substrate lattice mismatch is biaxial and hence it is parallel to the interface. ${ }^{49}$

The hexagonal symmetry of the InN/AlN(0001) system presumes that the shear stress is zero on the usual glide planes. $^{29,33,53}$ Consequently, the resolved shear stress on the (0001) basal planes (parallel to the interface), as well as that on the $\{1 \overline{1} 00\}$ prism planes (perpendicular to the interface) is zero, and it is unlikely that any of the dislocations lying on these planes are formed by the mismatch stresses. In addition, the "efficiency" of the MDs to relieve the biaxial stress is directly proportional to the edge component of the dislocation. In this way, the following slip systems: (a) $\{11 \overline{2} 2\}$ $\langle 11 \overline{2} 3\rangle$ and (b) $\{1 \overline{1} 01\}\langle 11 \overline{2} 3\rangle$ were found to be the only ones possible of forming SMDs, more and less probable, respectively, to relieve biaxial strain. ${ }^{33}$

\section{TDs in $2 H$-InN induced by coalescence}

Before the $2 H$-InN islands coalesce to form a continuous film, the nuclei are often faceted on low-energy basal, prismatic, and pyramidal planes forming a top truncated hexagonal-based pseudoprism/pyramid [see Figs. 1 and 4(c)]. Moreover, 3D nucleation and low temperatures of InN growth lead to the deposition of nuclei that are misoriented with respect to each other by small twist angles $\left(<0.5^{\circ}\right)$ in arbitrary crystallographic direction forming a partially disordered phase. ${ }^{49,54}$

During the coalescence, $2 \mathrm{H}$-InN domains come into contact. The small misorientation between domains will give rise to a low-angle domain boundary. As illustrated in Fig. 10, when two strained platelets are close together and, for example, their approximately parallel $\{1 \overline{1} 00\}$ planes come into contact, the distance between the edge atoms of each platelet is modified due to the edge relaxation. For platelets in tension, this distance is larger; for compressively strained it is smaller. Thus, it is favorable for adatoms to nucleate an edge dislocation with a positive (tensile strain) or negative (compressive strain) Burger's vector at the trough between two islands. ${ }^{16}$ The coexistence of hexagonal and cubic islands [see Figs. 8(a) and 8(b)] would further increase the probability to nucleate the appropriate positive /negative dislocation.

For the islands with similar $c$ orientation, the domain boundary will be a "pure tilt boundary" and the dislocation lines will be parallel to [0001] axis having pure edge character, with $\mathbf{b} \|\langle 11 \overline{2} 0\rangle$. A relative rotation around the $\langle 1 \overline{1} 00\rangle$ axis, parallel to the interface, induces a "pure twist boundary" causing formation of a network of orthogonal screw 
(a)
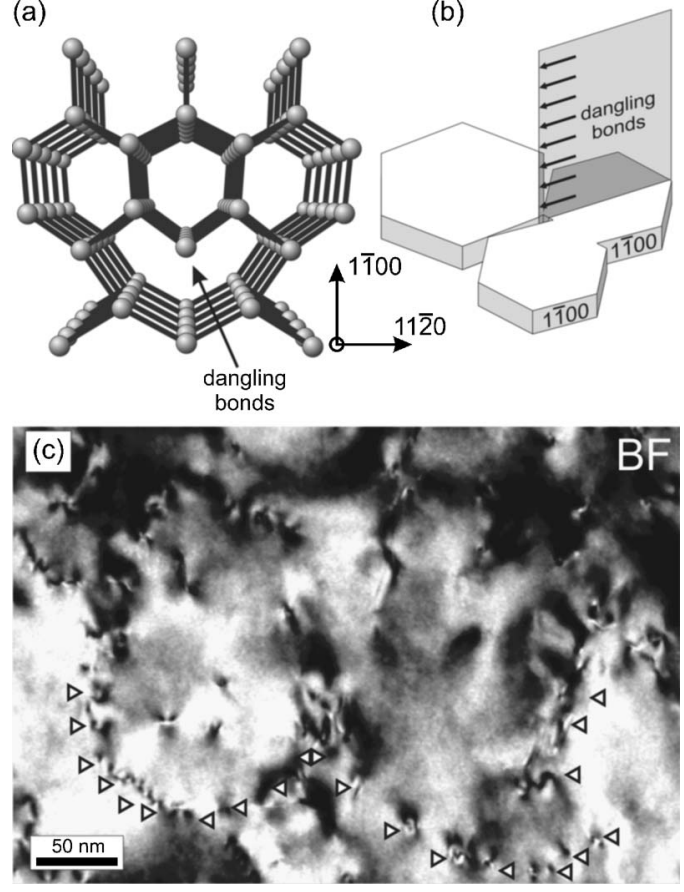

FIG. 10. (a) Full-core model of a threading edge dislocation in the wurtzite structure. (b) A schematic view of the grain boundary threading edge dislocation at the contact plane of the $2 \mathrm{H}$-InN islands. (c) BF PVTEM micrograph of a $70 \mathrm{~nm} 2 \mathrm{H}$-InN film taken in $2 \mathrm{~B}$ conditions obtained with the $(11 \overline{2} 0)$ reflection registered near the [0001] zone axis of InN. Dislocations reaching the InN surface, which are visible in these conditions, are edgetype $\mathbf{b}=1 / 3\langle 11 \overline{2} 0\rangle$ and $\bar{b}=1 / 3\langle 11 \overline{2} 3\rangle$ TDs. Some of the dislocations are marked by open triangles to highlight the contour of the grain boundary.

dislocations on the $\{1 \overline{1} 00\}$ boundary plane with one set parallel to the $c$ axis $(\mathbf{b} \|[0001])$ and the other set parallel to $\langle 11 \overline{2} 0\rangle(\mathbf{b} \|\langle 11 \overline{2} 0\rangle)$. In the actual crystal, due to the arbitrary misorientation of the domains during the nucleation phase, the boundaries of a mixed "tilt-twist" character are mainly formed after the coalescence phase. In this case, the dislocation network consists of sets of dislocations parallel to [0001] and to $\langle 11 \overline{2} 0\rangle$. Shown in Fig. 10(c), a PVTEM micrograph of a $70 \mathrm{~nm} 2 \mathrm{H}$-InN film taken in $2 \mathrm{~B}$ conditions illustrates formation of TDs at the grain boundaries showing a typical in-plane TD distribution.

The character of the dislocations depends also on the character of the domain boundary, and the spacing $D$ is related to the misorientation $\Delta \phi$ and the Burger's vector $\mathbf{b}$ by $D=|\mathbf{b}| / \tan (\Delta \phi)$. Thus, if we assume that $\mathbf{b}=1 / 3\langle 11 \overline{2} 0\rangle$ and $\Delta \phi \sim 0.3^{\circ}$ (as it follows from XRD measurements), then $D$ is $\sim 60 \mathrm{~nm}$ and the approximated dislocation density $D_{c} \approx 7.7$ $\times 10^{10} \mathrm{~cm}^{-2}$. The value of $D$ is in accordance with the approximate density of domains participating in the coalescence phase. A similar mechanism was also proposed for the $2 \mathrm{H}-\mathrm{GaN} / \mathrm{AlN} / \mathrm{Al}_{2} \mathrm{O}_{3}$ (Ref. 55) and for the $2 \mathrm{H}-\mathrm{GaN} / \mathrm{Al}_{2} \mathrm{O}_{3}$ (Ref. 49) structures, where $\Delta \phi$ was estimated to be as high as $\sim 3^{\circ}$ and $\sim 0.3^{\circ}$, respectively.

Since there is no appreciable resolved shear stress on some planes ${ }^{49}$ (e.g., the activation of prism plane dislocations is not very probable added to the fact that $\{1 \overline{1} 00\}$ or $\{11 \overline{2} 0\}$ are not operative by the characteristic of the current epitaxial growth), this dislocation network does not accommodate residual lattice-mismatch stress in the epilayer. Thus, the $|\mathbf{b}|=1 / 3\langle 11 \overline{2} 0\rangle$ edge dislocations are growth dislocations (with no dislocation glide) produced by the domain structure of the film which occur during the nucleation stage. It was also shown experimentally ${ }^{56,57}$ and by theoretical modeling ${ }^{58,59}$ that dislocations are formed at the corners of the islands, where the strain is mostly concentrated.

Obviously, the island growth as well as a dislocation formation process is influenced by the growth parameters, e.g., supersaturation and substrate temperature. It has been shown, for instance, that N-rich conditions lead to 3D nucleation and enhance the formation of stacking faults. ${ }^{60,61}$

\section{E. Elastic relaxation by the SMD network}

Even in the case of a sufficiently small misfit, as the layer thickness increases, the homogeneous strain energy $E_{\text {str }}$ becomes larger. It is emphasized that the correspondence between the $m$ planes of the film and $n$ planes of the substrate is only approximate. The residual mismatch is accommodated by an elastic strain in the epilayer giving a biaxial stress of

$$
\sigma_{x x}=2 G f \frac{1+\nu}{1-\nu} .
$$

At a certain critical thickness $d_{c},{ }^{48}$ it may become possible that the strain energy is partially released by generation of lattice dislocations (SMDs) which glide to the island/ substrate interface (this may also occur by the glide of preexisting lattice dislocations to the interface). The inward (outward) relaxation is proportional to the residual misfit $f$.

The density of SMDs directly depends on the biaxial strain remaining in the system after the coalescence phase. The variation of the biaxial strain $\mathfrak{I}_{x x}$ as a function of the continuous layer thickness $d$ can be described as

$$
\mathfrak{I}_{x x}(d)=\mathfrak{I}_{x x}^{0} \exp \left(-\frac{d-d_{c}}{\Lambda}\right),
$$

where $\mathfrak{I}_{x x}^{0}$ is the initial "dynamical" strain and $\Lambda \sim 10 \mathrm{~nm}$ is the characteristic thickness for the irreversible relaxation measured by RHEED. ${ }^{16}$ The residual biaxial strain can be related to the local misfit dislocations density $D_{S}(d)$ as follows:

$$
D_{S}(d)=\frac{\Im_{x x}^{0}}{b_{\perp}}\left[1-\exp \left(-\frac{d-d_{c}}{\Lambda}\right)\right],
$$

where $b_{\perp}$ is the Burger's vector component parallel to the interface and perpendicular to the dislocation line. From Eq. (9), we can deduce an approximate SMD density that grows with the thickness to release the increase of elastic misfit energy. This does not imply that, as shown previously, some of these related SMD segments will not be placed in the near interface region.

The basic assumption of the elastic relaxation theory ${ }^{41-43}$ is that the configuration of the episystem is the one of minimum energy. In other words, the actual configuration of the considered system described by Eq. (4) will be given by the 
strain for which $E_{\mathrm{str}}=E_{\mathrm{str}}(\mathfrak{I})$ is a minimum. For a given thickness the minimum energy occurs for a value $\mathfrak{I}_{0}$ given by

$$
\mathfrak{I}_{0}=\frac{b}{8 \pi d(1+\nu)} \ln \left(\frac{e d}{r_{0}}\right) .
$$

If $\mathfrak{I}_{0}<f$, then some misfits will be relaxed by dislocations with $\mathfrak{I}_{0}=f-b / p$. The critical film thickness, $d_{c}$, at which the creation SMDs become energetically favorable is obtained when $\mathfrak{I}_{0}=f$, i.e.,

$$
d_{c}=\frac{b \cdot\left(1-\cos ^{2} \Theta\right)}{8 \pi f(1+\nu) \sin \lambda \sin \Theta}\left[\ln \left(\frac{d_{c}}{r_{0}}\right)+1\right],
$$

with $\Theta$ being the angle between the dislocation line and its Burger's vector, and $\lambda$ as the angle between the slip direction and a line in the interface plane that is normal to the line of intersection between the slip plane and the interface.

As commented, we assume that mixed dislocations along the three equivalent $\langle 01 \overline{1} 0\rangle$ directions that glide at $\{11 \overline{2} 2\}$ planes with Burger's vector components parallel to $\langle 11 \overline{2} 3\rangle$ are able to relax biaxial strain. With the residual substrateepilayer misfit of $f=-0.0125$, a Burger's vector b $=1 / 3\langle 11 \overline{2} 3\rangle$, and $\{112 \overline{2}\}$ or $\{1 \overline{1} 01\}$ glide planes, the value of $d_{c}$ for the appearance of SMDs due to the residual biaxial stress in the epilayer can be estimated as $d_{c} \cong 7.5 \mathrm{~nm}$. However, due to the fact that the calculated value of $d_{c}$ depends on the assumed dislocation arrangement, only an estimation can be performed.

Moreover, the exact value $d_{c}$ cannot be either confirmed by or derived from the TEM analyses due to the introduction of a high density of TDs induced by the coalescence phase, and a possible later placement of SMDs in the near interface region. However, TEM observations show a local maxima at the TD distribution at a distance of $\sim 10 \mathrm{~nm}$ from the InN/AlN interface (see Fig. 11), which is similar to the expected value of the critical thickness.

If we assume that each SMD could be related at least with one secondary TD and its length is limited by the mosaic structure, a secondary planar TD density is deduced by

$$
D_{2}(d)=\frac{8 \sqrt{3}}{L} D_{s}(d) .
$$

In this context, according to XRD measurements, our layers are fully relaxed after $350 \mathrm{~nm}$ of growth. From Eq. (12) a density of secondary TDs of $D_{2} \sim 3.26 \times 10^{11} \mathrm{~cm}^{-2}$ is estimated.

\section{F. Total density of TDs}

Finally, we can estimate the total density of TDs in the epitaxial $2 H$-InN film grown on AlN templates after the coalescence phase as follows:

$$
D=D_{t}+D_{c}(\Im)+D_{2}(\Im) .
$$

GMDs have not been taken into account, so as to not create dislocation lines propagating along the $\langle 0001\rangle$ direction. The calculated total density of TDs, $D \sim 4.07$ $\times 10^{11} \mathrm{~cm}^{-2}$, fits well to the value obtained by the TEM observations: for dislocations with pure edge or mix character
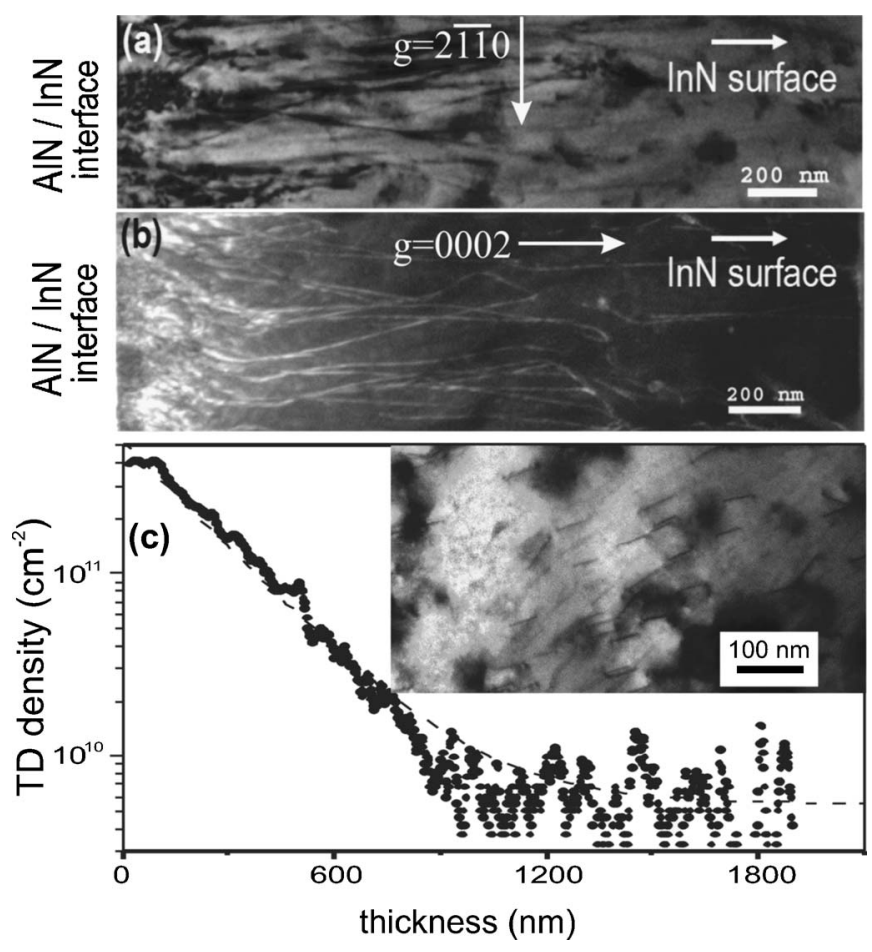

FIG. 11. (a) BF-TEM micrograph taken in $2 \mathrm{~B}$ conditions using the $11 \overline{2} 0$ reflection; using these conditions, pure edge $\mathbf{b}=1 / 3\langle 11 \overline{2} 0\rangle$ or mixed $\bar{b}$ $=1 / 3\langle 11 \overline{2} 3\rangle$ TDs are highlighted. (b) DF-TEM micrograph taken in $2 \mathrm{~B}$ conditions using the 0002 reflection where pure screw $\mathbf{b}=(0001)$ or mix $\mathbf{b}$ $=1 / 3\langle 11 \overline{2} 3\rangle$ TDs are easily visible. (c) Edge TD density vs distance from the interface for $2.2 \mu \mathrm{m}$ thick $\mathrm{InN}$ epilayer. The plot combines the data derived from the XTEM studies; inset: PVTEM micrograph taken in 2B conditions shows TDs reaching the $\mathrm{InN}$ surface.

at a thickness of $\sim 7.5 \mathrm{~nm}$ the experimental value is 3 $\times 10^{11} \mathrm{~cm}^{-2}$, and the extrapolated value is $4 \times 10^{11} \mathrm{~cm}^{-2}$ (see Fig. 11).

Despite the fact that a reasonable correspondence between calculated and measured values has been reached, some remarks concerning the applicability of the "critical thickness" approach have to be made. Firstly, it is generally found that the observed values of $d_{c}$ differ significantly from those predicted over the large range of misfits in the III-N heterostructures. ${ }^{19,23,25,62}$ In nonequilibrium conditions characteristic for PIMBE, experimental values are very dependent on the growth conditions, and very often, the strain relief is limited by the growth kinetic. As a result, dislocations are introduced with no well-defined critical thickness. The rate of introduction is also dependent on the stoichiometry ratio and, as a consequence, on the surface roughness. ${ }^{16,49}$

From the practical point of view, since the growth mode in a large lattice-mismatched system involves formation of 3D nuclei and their growth and coalescence into a continuous film, a more appropriate criterion is a "critical size" for the nucleated domains rather than "critical thickness" for the continuous film. Especially, in the case of low-temperature PIMBE of InN, the temperature can be low enough preventing effective dislocation generation in the film. Consequently, residual strains and stresses will remain in the film resulting in the characteristic $2 \mathrm{D} \rightarrow 3 \mathrm{D}$ transition for thicker 
films leading to the pure Wolmer-Weber mode of growth. The experimentally observed value for this "secondary critical thickness" is $\sim 1.6 \mu \mathrm{m}$ for the $2 \mathrm{H}$-InN on AlN templates grown at $370{ }^{\circ} \mathrm{C}$. In this case, the dislocation density in the film bulk is determined by the average size of the domains during the coalescence phase and the second term in Eq. (4) plays the dominant role.

\section{G. Elimination of dislocations}

Figure 11(a) shows a bright-field cross-sectional transmission electron microscopy (XTEM) micrograph taken in two beam conditions using the $11 \overline{2} 0$ reflection of $\mathrm{InN}$ along the $[1 \overline{1} 00]$ zone axis. The inset in Fig. 11(c) shows a brightfield PVTEM image in two beam conditions using the $11 \overline{2} 0$ reflection along the [0001] zone axis. In both cases, under these conditions, TDs with an edge component b $=1 / 3\langle 11 \overline{2} 0\rangle$ are displayed; i.e., TDs with pure edge: $\mathbf{b}=\mathbf{a}$ or mixed $\mathbf{b}=(\mathbf{a}+\mathbf{c})$ character. The reflection used for the DF image of Fig. 11(b) is better in order to obtain highly contrasted images of TDs since the product $\mathbf{g} \cdot \mathbf{b}$ in $2 \mathrm{~B}$ conditions for wurtzite systems is maximized. On the other hand, these TDs have a pure screw: $\mathbf{b}=\mathbf{c}$ or mixed: $\mathbf{b}=(\mathbf{a}+\mathbf{c})$ character. Our measurements from XTEM and PVTEM micrographs on the InN surface of a $2.2 \mu \mathrm{m}$ layer allow us to estimate a density relationship as $\varphi[a+(a+c)]: \sim 3 \times 10^{9} \mathrm{~cm}^{-2} / \varphi[c$ $+(a+c)]: \sim 1 \times 10^{9} \mathrm{~cm}^{-2}$. In fact, it is described by other studies $^{29}$ that the most common relation between $[a+(a$ $+c)] /[c+(a+c)]$ TDs on GaN epilayers is $90 / 40$. We have also used the intensity profile of Fig. 11(b) in order to calculate how TDs of every type are being eliminated as the thickness increases. Using the data of TD densities measured at the InN surface and considering that both groups of dislocations have a similar tendency to annihilate, ${ }^{29}$ we can extrapolate values at different thicknesses from Fig. 11(c). For example, $\varphi[a+(a+c)] \sim 4 \times 10^{11} \mathrm{~cm}^{-2}$ can be derived for $d_{c}$ $\sim 7.5 \mathrm{~nm}$. To compare, $\varphi[a+(a+c)] \sim 7 \times 10^{11} \mathrm{~cm}^{-2}$ was reported for $2.4 \%$ mismatched $\mathrm{AlN} / \mathrm{GaN}$ heterosystems. ${ }^{16}$

As one can see in Fig. 11(c), the reduction in the dislocation density follows an exponential decay law up to the thickness of $\sim 1 \mu \mathrm{m}$. The corresponding reduction in dislocation density $\left(N_{D}\right)$ exceeds two orders of magnitude with $N_{D} \sim 1 \times 10^{10} \mathrm{~cm}^{-2}$ for a $1 \mu \mathrm{m}$ thick InN. However, a large decrease in the TD density is not observed after these thicknesses. It was observed many times by TEM studies that the TD density in the epilayer decreases near exponentially with the layer thickness (see, for instance, Refs. 16 and 63). The exponential decay can be explained by the fact that TD annihilation probability depends directly on the density of the dislocation lines, namely, $\mathbf{b}=1 / 3\langle 11 \overline{2} 0\rangle$ threading dislocations, DBs, and $\mathbf{b}=(0001)$ stacking faults $(\mathrm{SFs})$. Moreover, remaining compressive strain after the coalescence phase is expected to enable much more rapid threading dislocation motion and cause improved dislocation annihilation kinetics. $^{44}$

The main mechanism of TD annihilation in $2 \mathrm{H}$-InN is similar to those observed in $2 \mathrm{H}$-GaN (Ref. 64) and $2 \mathrm{H}$ AlN: ${ }^{15}$ two TDs in InN can form a half loop by means of introducing a dislocation segment along and parallel to the (0001) basal plane [Fig. 8(d), selection 1]. Randomly distributed SFs lying parallel to the interface plane are also peculiar for the wurtzite epilayers. These planar defects are also responsible for the reduction of the TD density, being efficient traps for bending or stacking the TD propagation [Fig. 8(d), selection 2].

The $\mathbf{b}=[0001]$ dislocation half loops lying on the $\{1 \overline{100}\}$ prism plane and $\mathbf{b}=1 / 3\langle 11 \overline{2} 0\rangle$ dislocation half loops lying on the (0001) basal planes near the film/substrate interface have a relatively low density of $\sim 10^{8} \mathrm{~cm}^{-2}$. However, this kind of dislocation plays an important role in the elimination of threading dislocations in the epilayer.

\section{CONCLUSION}

The strain-relaxation phenomena and formation of the dislocation network in $2 \mathrm{H}$-InN epilayers during PIMBE have been discussed. In the framework of a growth model, subsequent stages of the strain relief have been proposed: plastic relaxation of the primary strain by the introduction of geometric misfit dislocations, elastic strain relief during the island growth phase, formation of threading dislocations induced by the coalescence of the domains, and relaxation of the elastic strain by the introduction of secondary misfit dislocations. The model emphasizes the determinant role of the coalescence process in the formation of a network of dislocations in heteroepitaxially grown $2 \mathrm{H}$-InN as revealed by RHEED, TEM, and XRD measurements.

The density and types of dislocations were characterized by TEM. Edge type $\mathbf{b}=1 / 3\langle 11 \overline{2} 0\rangle$ and TDs of mixed character have been found to be the main defects in wurtzite InN layers. It has been shown that the TD density decreases exponentially during the film growth due to annihilation and fusion of the dislocations reaching $\sim 1-3 \times 10^{9} \mathrm{~cm}^{-2}$ for an InN thickness of $\sim 2.2 \mu \mathrm{m}$. Photoelectron spectroscopy revealed that careful annealing at $500 \mathrm{~K}$ results in a deoxidation of the surface together with a better appearance of the real $\mathrm{InN}$ electronic properties.

The effect of the dislocation network on electron transport properties of nominally undoped $2 H$-InN thin films is reported in the second part of this work, where direct dependence between the carrier generation and the dislocation density in $2 \mathrm{H}$-InN is considered.

\section{ACKNOWLEDGMENTS}

This work was supported by the European Union (the sixth framework program: NMP4-CT2003-505614 and NMP4-CT-2004-500101) and CICYT Project No. MAT2004-01234 (Spain). One of the authors (F.M.M.) would like to thank the Alexander von Humboldt Foundation for the financial support under a Humboldt Research Fellowship SPA/1114640STP. The authors would like to thank A. Williamson for his help in preparing the manuscript.

\footnotetext{
${ }^{1}$ V. Davydov et al., Phys. Status Solidi B 229, R1 (2002); 230, R4 (2002). ${ }^{2}$ J. Wu et al., Appl. Phys. Lett. 80, 3967 (2002).

${ }^{3}$ T. Matsuoka, H. Okamoto, M. Nakao, H. Harima, and E. Kurimoto, Appl. Phys. Lett. 81, 1246 (2002).
} 
${ }^{4}$ H. Lu, W. J. Schaff, L. F. Eastman, and C. E. Stutz, Appl. Phys. Lett. 82, 1736 (2003).

${ }^{5}$ A. G. Bhuiyan, A. Hashimoto, and A. Yamamoto, J. Appl. Phys. 94, 2779 (2003).

${ }^{6}$ S. N. Mohammad and H. Morkoc, Prog. Quantum Electron. 20, 361 (1996).

${ }^{7}$ V. W. L. Chin, T. L. Tansley, and T. Osotchan, J. Appl. Phys. 75, 7365 (1994).

${ }^{8}$ S. K. O'Leary, B. E. Foutz, M. S. Shur, U. V. Bhapkar, and L. F. Eastman, J. Appl. Phys. 83, 826 (1998).

${ }^{9}$ B. E. Foutz, S. K. O'Leary, M. S. Shur, and L. F. Eastman, J. Appl. Phys. 85, 7727 (1999).

${ }^{10}$ H. Lu, W. J. Schaff, and L. F. Eastman, J. Appl. Phys. 96, 3577 (2004).

${ }^{11}$ E. Bellet-Amalric, C. Adelmann, E. Sarigiannidou, J. L. Rouvière, G. Feuillet, E. Monroy, and B. Daudin, J. Appl. Phys. 95, 1127 (2004).

${ }^{12}$ S. J. Rosner, E. C. Carr, M. J. Ludowise, G. Girolami, and H. I. Erikson, Appl. Phys. Lett. 70, 420 (1997).

${ }^{13}$ T. Sugahara et al., Jpn. J. Appl. Phys., Part 2 37, L398 (1998).

${ }^{14}$ N. G. Weimann, L. F. Eastman, D. Doppalapudi, H. M. Ng, and T. D. Moustakas, J. Appl. Phys. 83, 3656 (1998).

${ }^{15}$ V. Lebedev et al., J. Appl. Phys. 98, 093508 (2005).

${ }^{16}$ A. Bourret, C. Adelmann, B. Daudin, J. Rouvière, G. Feuillet, and G. Mula, Phys. Rev. B 63, 245307 (2001).

${ }^{17}$ S. Hearne, E. Chason, J. Han, J. A. Floro, J. Figiel, J. Hunter, H. Amano, and I. S. T. Tsong, Appl. Phys. Lett. 74, 356 (1999).

${ }^{18}$ A. V. Dobrynin, J. Appl. Phys. 85, 1876 (1999).

${ }^{19}$ C. Kim, I. K. Robinson, J. Myoung, K.-H. Shim, and K. Kim, J. Appl. Phys. 85, 4040 (1999)

${ }^{20}$ A. R. Woll, R. L. Headrick, S. Kycia, and J. D. Brock, Phys. Rev. Lett. 83, 4349 (1999).

${ }^{21}$ D. C. Reynolds, D. C. Look, B. Jogai, J. E. Hoelscher, R. E. Sherriff, and R. J. Molnar, J. Appl. Phys. 88, 1460 (2000).

${ }^{22}$ A. D. Bykhovski, B. L. Gelmont, and M. S. Shur, J. Appl. Phys. 78, 3691 (1995).

${ }^{23}$ N. Grandjean and J. Massies, Appl. Phys. Lett. 71, 1816 (1997).

${ }^{24}$ G. Feuillet, B. Daudin, F. Widmann, J. L. Rouviere, and M. Arlery, J. Cryst. Growth 190, 142 (1998).

${ }^{25}$ R. Langer, A. Barski, A. Barbier, G. Renaud, M. Leszczynski, I. Grzegory, and S. Porowski, J. Cryst. Growth 205, 31 (1999).

${ }^{26}$ R. Langer, A. Barski, J. Simon, N. T. Pelekanos, O. Konovalov, R. Andre, and L. S. Dang, Appl. Phys. Lett. 74, 3610 (1999).

${ }^{27}$ X. H. Wu, P. Fini, E. J. Tarsa, B. Heying, S. Keller, U. K. Mishra, S. P. DenBaars, and J. S. Speck, J. Cryst. Growth 190, 231 (1998).

${ }^{28}$ R. L. Headrick, S. Kycia, A. R. Woll, J. D. Brock, and M. V. Ramana Murty, Phys. Rev. B 58, 4818 (1998).

${ }^{29}$ S. Mathis, A. Romanov, L. Chen, G. Beltz, W. Pompe, and J. Speck, J. Cryst. Growth 231, 371 (2001).

${ }^{30}$ Y. F. Ng, Y. G. Cao, M. H. Xie, X. L. Wang, and S. Y. Tong, Appl. Phys. Lett. 81, 3960 (2002).

${ }^{31}$ V. Cimalla et al., Phys. Status Solidi C 0, 2818 (2003).

${ }^{32}$ Y. G. Cao, M. H. Xie, Y. Liu, Y. F. Ng, H. S. Wu, and S. Y. Tong, Appl. Phys. Lett. 83, 5157 (2003).
${ }^{33}$ S. Srinivasan, L. Geng, R. Liu, F. A. Ponce, Y. Narukawa, and S. Tanaka, Appl. Phys. Lett. 83, 5187 (2003).

${ }^{34}$ F. M. Morales, S. I. Molina, D. Araujo, R. García, V. Cimalla, and J. Pezoldt, Diamond Relat. Mater. 12, 1227 (2003).

${ }^{35}$ V. Lebedev, V. Cimalla, U. Kaiser, Ch. Foerster, J. Pezoldt, J. Biskupek, and O. Ambacher, J. Appl. Phys. 97, 114306 (2005).

${ }^{36}$ S. Krischok et al., Surf. Sci. 566-568, 849 (2004).

${ }^{37}$ A. F. Wright, J. Appl. Phys. 82, 2833 (1997).

${ }^{38}$ Y. Bu, L. Ma, and M. C. Lin, J. Vac. Sci. Technol. A 11, 2931 (1993).

${ }^{39}$ L. F. J. Piper et al., Phys. Rev. B 72, 245319 (2005).

${ }^{40}$ J. Howe, Interfaces in Materials (Wiley, New York, 1997).

${ }^{41}$ J. H. Van Der Merwe, J. Appl. Phys. 34, 123 (1963); J. Appl. Phys. 34, 117 (1963)

${ }^{42}$ J. Matthews and A. Blakeslee, J. Cryst. Growth 27, 18 (1974); 29, 273 (1975); 32, 265 (1976).

${ }^{43}$ J. Matthews and A. Blakeslee, J. Vac. Sci. Technol. 14, 989 (1977).

${ }^{44}$ P. J. Taylor et al., J. Appl. Phys. 89, 4365 (2001).

${ }^{45}$ E. Bellet-Amalric, C. Adelmann, E. Sarigiannidou, J. L. Rouvière, G. Feuillet, E. Monroy, and B. Daudin J. Appl. Phys. 95, 1127 (2004).

${ }^{46}$ V. Lebedev, B. Schröter, G. Kipshidze, and W. Richter, J. Cryst. Growth 207, 266 (1999).

${ }^{47}$ V. Lebedev, J. Jinschek, U. Kaiser, B. Schröter, W. Richter, and J. Kräußlich, Appl. Phys. Lett. 76, 2029 (2000).

${ }^{48}$ F. Frank and J. van der Merwe, Proc. R. Soc. London, Ser. A 198, 205 (1949).

${ }^{49}$ X. J. Ning, F. R. Chien, P. Pirouz, J. W. Yang, and M. Asif Khan, J. Mater. Res. 11, 580 (1996).

${ }^{50}$ P. Cantu, F. Wu, P. Waltereit, S. Keller, A. E. Romanov, U. K. Mishra, S. P. DenBaars, and J. S. Speck, Appl. Phys. Lett. 83, 674 (2003).

${ }^{51}$ R. Kern and P. Müller, Surf. Sci. 392, 103 (1997).

${ }^{52}$ J. P. Hirth and J. Lothe, Theory of Dislocations, 2nd ed. (Wiley, New York, 1982).

${ }^{53}$ V. Audurier, J. L. Demenet, and J. Rabier, Philos. Mag. A 77, 825 (1998).

${ }^{54}$ I. Akasaki, H. Amano, Y. Koide, K. Hiramatsu, and N. Sawaki, J. Cryst. Growth 98, 209 (1989).

${ }^{55}$ W. Qian, M. Skowronski, M. De Graaf, K. Doverspike, L. B. Rowland, and D. K. Gaskill, Appl. Phys. Lett. 66, 1252 (1995).

${ }^{56}$ F. K. LeGoues, M. C. Reuter, J. Tersoff, M. Hammar, and R. M. Tromp, Phys. Rev. Lett. 73, 300 (1994); Appl. Phys. Lett. 67, 2317 (1995).

${ }^{57}$ Y. Chen, X. W. Lin, Z. Lilienthal-Weber, J. Washburn, J. F. Klemm, and J. Y. Tsao, Appl. Phys. Lett. 68, 111 (1996).

${ }^{58}$ H. T. Johnson and L. B. Freud, J. Appl. Phys. 81, 6081 (1997).

${ }^{59}$ B. J. Spencer and J. Tersoff, Appl. Phys. Lett. 77, 2533 (2000).

${ }^{60}$ E. J. Tarsa, B. Heying, X. H. Wu, P. Fini, S. P. DenBaars, and J. S. Speck, J. Appl. Phys. 82, 5472 (1997).

${ }^{61}$ F. Widmann, B. Daudin, G. Feuillet, N. Pelekanos, and J. L. Rouviere, Appl. Phys. Lett. 73, 2642 (1998).

${ }^{62}$ Z. Sitar, M. J. Paisley, B. Yan, J. Ruan, W. J. Choyke, and R. F. Davis, J. Vac. Sci. Technol. B 8, 316 (1990).

${ }^{63}$ J. E. Dominguez, L. Fu, and X. Q. Pan, Appl. Phys. Lett. 81, 5168 (2002).

${ }^{64}$ S. Tanaka, M. Takeuchi, and Y. Aoyagi, Jpn. J. Appl. Phys., Part 1 39, L831 (2000). 